Development of Intertwined Infills to Improve Multi-Material Interfacial Bond Strength

\author{
Irfan Mustafa
}

A Thesis

in

The Department

of

Mechanical, Industrial and Aerospace Engineering

Presented in Partial Fulfillment of the Requirements

for the Degree of Master of Applied Science (Mechanical Engineering) at

Concordia University

Montreal, Quebec, Canada

April 2021

(C) Irfan Mustafa, 2021 


\section{CONCORDIA UNIVERSITY}

\section{School of Graduate Studies}

This is to certify that the thesis prepared

By: $\quad$ Irfan Mustafa

Entitled: Development of Intertwined Infills to Improve Multi-Material Interfacial Bond Strength

and submitted in partial fulfillment of the requirements for the degree of

\section{Master of Applied Science (Mechanical Engineering)}

complies with the regulations of the University and meets the accepted standards with respect to originality and quality.

Signed by the final Examining Committee:

Chair \& Examiner

Dr. Martin D. Pugh

Examiner

Dr. Chevy Chen

Supervisor

Dr. Tsz Ho Kwok

Approved by

Dr. Martin D. Pugh, Chair of Department or Graduate Program Director

Date

Dr. Mourad Debbabi, Interim Dean of Gina Cody School of Engineering

and Computer Science 


\section{Abstract \\ Development of Intertwined Infills to Improve Multi-Material Interfacial Bond Strength \\ by Irfan Mustafa}

Multi-material additive-manufacturing (MMAM) technology provides a solution to 3D print a variety of parts consisting of multiple materials without the necessity of performing complex manufacturing processes. Till now various MMAM techniques have been developed for different applications. The wide availability of materials and recent developments in MMAM has opened doors for innovation in producing truly functional products using various materials. A lot of research is research going on in developing efficient 3D printers for MMAM, but conventional software tools does not full-fill the modern requirements suited for 3D printing of multi-material structures. There is an existing research gap between existing CAD tools, 3D printers and slicing software 3D printing of multi-material parts. One major concern of MMAM is the strength at the interface between materials. Based on the observation of how nature puts materials together, this research develops an initial hypothesis that if the materials are overlapped and interlaced with each other, the interface bonding strength will be enhanced. To test this hypothesis, a computer-aided manufacturing (CAM) tool that can process overlapped material regions is needed. However, existing computational tools lack key multi-material design processing features and have certain limitations in making full use of material information, which restricts the test of the hypothesis.

Therefore, this research also develops a new MMAM slicing framework that efficiently identifies the multi-material regions and develops interlaced infills. Based on ray-tracing technology, layered depth material images (LDMI) is developed to process 
the material information from computer-aided design (CAD) models for tool-path planning. Each sample point in the LDMI has an associated material and geometric properties that are used to recover the material distribution in each slice. In this research, an interlocking joint (T-joint) and an interlacing infill are developed in the regions with multiple materials. By carrying out tensile tests, it is shown that the proposed infill outperforms the interlocking joint, and a fracture occurs even outside the joint area. This validates the initial hypothesis, and the enhancement of interface strength is achieved by overlapping and interlacing materials. 


\section{Acknowledgements}

I am most thankful to Almighty Allah for providing me the opportunity to pursue my graduate studies and research and enabling me to complete the Master of Applied Science (Mechanical Engineering) program at Concordia University. I am thankful to my advisor Tsz Ho Kwok, who recognized my potential as a scholar and opened a pathway for me to become one. Your persistent guidance and support, particularly at difficult points in my research, are the reasons why I have finally arrived at the end of the MASc. endeavour. Moreover, your passionate and shrewd comments on the countless drafts of the research paper and thesis nurtured me to think and write critically as a researcher. I would also like to thank my committee members, Dr Martin D. Pugh and Dr Chevy Chen for their incredibly helpful and indispensable comments and advice. My lab mates in the Design for Additive Manufacturing have been great teammates, collaborators, and friends who always made time to answer my questions and discuss research problems with me Finally, I would like to thank my parent for their unwavering support and unconditional support towards my MASc pursuit. Thank you for providing me with the opportunities and support to pursue the MASc program. Your unconditional love has helped me in every aspect to complete this pursuit and further forge future pathways. 


\section{Contents}

List of Figures

\begin{tabular}{|ll|}
\hline 1 & Introduction, Motivation \& Objectives
\end{tabular}

1.1 Scope of the Research Proposal . . . . . . . . . . . . . . . . . . . 1

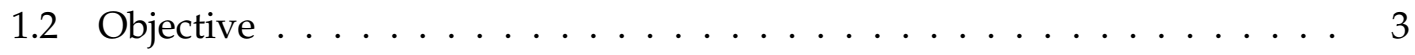

1.3 Major Contributions $\ldots \ldots \ldots \ldots \ldots \ldots$

1.4 Outline of the thesis $\ldots \ldots \ldots \ldots \ldots$

\begin{tabular}{lll}
\hline 2 & Literature Review & 7
\end{tabular}

2.1 Multi-material additive manufacturing $\ldots \ldots \ldots \ldots$

2.2 Process planning for multi-material printing $\ldots \ldots \ldots \ldots$

2.3 Interface and bonding between materials $\ldots \ldots \ldots$. . . . . . . . 10

2.4 Summary . . . . . . . . . . . . . . . . . . . . 12

\begin{tabular}{lll}
\hline & Methodology & 13
\end{tabular}

$3.1 \quad$ Ray-tracing with material information $\ldots \ldots \ldots$. . . . . . . . . 15

3.2 Slicing for multiple materials . . . . . . . . . . . . . . . . . . 19

Determining materials for grid nodes . . . . . . . . . . . 20

Forming material regions . . . . . . . . . . . . . 23

3.3 Modeling multi-material interfaces $\ldots \ldots \ldots \ldots$. . . . . . . . . 24

Interlaced infills . . . . . . . . . . . . . . . . 24

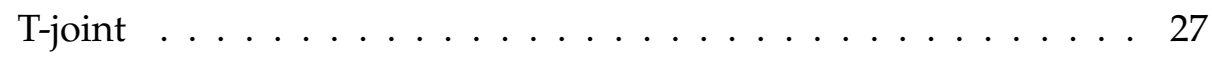

3.4 Summary . . . . . . . . . . . . . . . . . . . . . . . . 29 
\begin{tabular}{lll}
\hline 4 & Results & 30
\end{tabular}

4.1 Joint configurations . . . . . . . . . . . . . . . . . 32

4.2 Materials with distinct properties . . . . . . . . . . . . . . . . 34

4.3 Overlap size and interface strength $\ldots \ldots \ldots$. . . . . . . . . 36

5 Conclusion and Future Work 39

\begin{tabular}{ll}
\hline Bibliography & 41
\end{tabular} 


\section{List of Figures}

1.1 General steps in additive manufacturing . . . . . . . . . . . . . . . 2

1.2 Examples of human and nature intertwining materials: (a) Jute fibers are interlaced to form a tenacious cloth. (b) The lamellar microstructures of a Bi-Sn system are composed of alternating layers of bismuth (white) and tin (black) (C2003 by Charles and Cockburn (CC BY-NC-SA 2.0 UK). . . . . 4

1.3 (a) A model having three overlapping cylinders (top view) is inputted in a commercial slicer - Ultimaker Cura. It either (b) lets one infill take over the overlapping region or (c) makes the region solid. . . . . . . . . . . 5

$2.1 \quad$ Additive manufacturing technologies and applications of multi-material additive manufacturing $[41] @ 2020$ by Elsevier. $\ldots \ldots \ldots$. . . . . 8

2.2 Types of Interface analyzed by Ribeiro et al. [12]. (a) T-shape (b) U-shape (c) Dovetail shape $\ldots . . \ldots \ldots$. . . . . . . . . . . . . . 10

$3.1 \quad$ The workflow of the LDMI framework for processing and modeling multimaterial interface. . . . . . . . . . . . . . . . . . . 14

3.2 Ray tracing schematic diagram. . . . . . . . . . . . . . 16

3.3 Ray tracing process to obtain the LDMI sample points. . . . . . . . . . . 17

3.4 Slicing plane with classified gridnodes and material regions. . . . . . . . . 20

3.5 Determining materials for grid-nodes by LDMI sample points. Note that the $z$-axis is pointing to the right. . . . . . . . . . . . . . 21

3.6 Development of contour lines in cells with ' $\mathrm{G}$ ' representing grid-nodes on a slicing plane. . . . . . . . . . . . . . . . 24 
viii

3.7 Visualization of interlaced infills on a model of three overlapping cylinders with materials $A, B$, and $C$. . . . . . . . . . . . . 26

$3.8 \quad$ Infill shift parameter applied to materials $A, B$, and $C$ alternatively in subsequent layers to enhance interlacing effect. . . . . . . . . . . . . . . . . 27

3.9 Development of T-joints at the interface. . . . . . . . . . . . . . . . . . 29

4.1 The dog-bone samples for tensile test. . . . . . . . . . . . . . 30

4.2 The experimental setup for tensile test. . . . . . . . . . . . . . . . 31

4.3 Tensile test data for single-material samples. . . . . . . . . . . . . 32

4.4 Tensile test data for different joint configurations. $\ldots \ldots \ldots$. . . . . 33

$4.5 \quad$ Fractured samples of butt joint, T-joint, and interlaced infill. . . . . . . . . 34

$4.6 \quad$ Tensile test data for different material combinations and joint configurations. . . . . . . . . . . . . . . . . . . 35

$4.7 \quad$ Fractured samples of butt joints and interlaced infills for the material combinations: PLA-ASA and PLA-PETG. . . . . . . . . . . . . . . . 36

4.8 Tensile test data for different sizes of overlap. . . . . . . . . . . . . . 37

4.9 Fractured samples of interlaced infills with different overlaps: $5 \mathrm{~mm}$, $15 \mathrm{~mm}$, and $20 \mathrm{~mm} . \ldots \ldots \ldots \ldots \ldots$ 


\section{Introduction, Motivation \&}

\section{Objectives}

\subsection{Scope of the Research Proposal}

Throughout the years, Additive manufacturing (AM) technologies have been used for developing different prototypes and AM is a technology that is rapidly evolving and is continuously being integrated with the traditional manufacturing technologies in the

production of commercial products in various fields including automotive and aerospace. AM is being widely used in fabrication of real world applications and is possible using many different technologies and among different AM methods. Fused Filament Fabrication (FFF) [1] is one of the most extensively used AM and 3D printing process due to several reason including ease of use of technology. FFF 3D printers are the most widespread in the world. Additive manufacturing (AM) is a technology that builds 3D physical objects from digital 3D models and it utilizes a layer-by-layer stacking method to produce real three-dimensional (3D) structures from input geometry. Using layer-bylayer manufacturing method allows a great degree of freedom in manufacturing complex parts with a lot of precision and control which is not possible with traditional manufacturing methods [2, 3]. The general steps involved in the additive manufacturing process is shown in Fig. 1.1 Since their introduction more than 20 years ago, AM systems have been used in a variety of applications. These applications have ranged from conventional products to highly complex design models required in advanced applications. The geometric freedom and the free-form manufacturing technology enable AM 


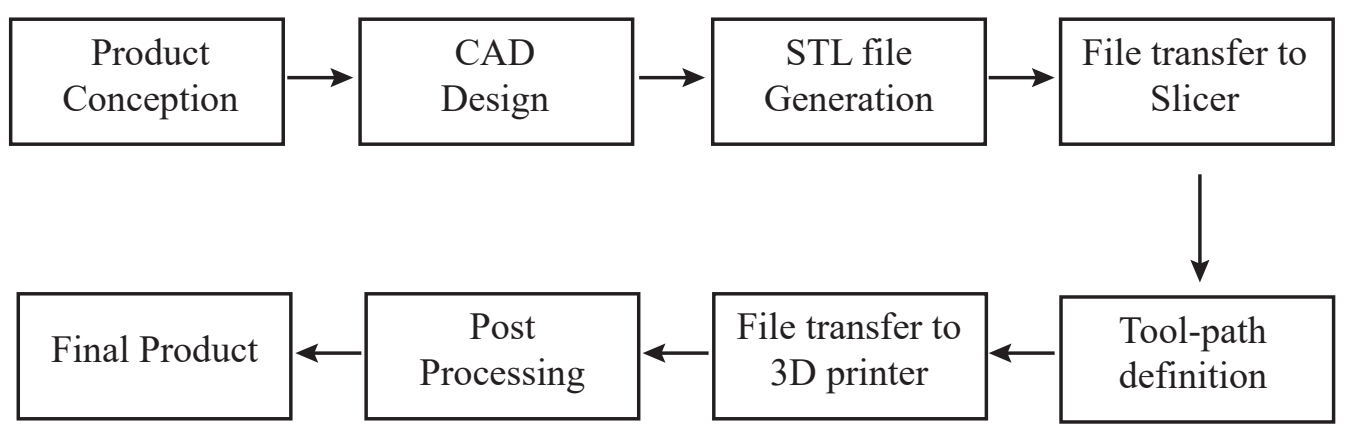

FIGURE 1.1: General steps in additive manufacturing

to fabricate complex structures with accurate material deposition capability at the specified positions defined in the design domain or computer-aided design (CAD) model. Research advances in AM and the availability of new materials have unlocked a new world of endless possibilities with customization in additive manufacturing that lead to complex multi-material structures [4, 5]. In the beginning of FFF evolution, the printing potential was limited to a single material with a small selection of available filament materials. With time and the continuous increase in the availability of different variety filament materials, FFF achieved a degree of flexibility in form of different available materials [6]. This enabled the development of different complex multi-material objects with a wide range of mechanical, physical and electronic properties. Over the years interest in the FFF technology has been greatly increasing within a number of industrial sectors due to its ability to easily fabricate complex structures with a variety of engineering materials and with less material waste. Well, it is challenging to predict how additive manufacturing will advance in the coming years but certainly, multi-material 3D printing is one way forward to develop multi-material structures with the various material combination (Metal, Polymer and Ceramic) [7, 8]. A multi-material object is a solid model composed of varying material properties within a specified domain inside the geometry. The availability of diverse materials with different properties is one of the main reasons for innovation happening in the development of multi-material structures. This allows overcoming the existing single material AM limitations for the development of 
functional objects. Multi-material additive manufacturing (MMAM) has a lot of benefits and that is why MMAM technology is innovating rapidly to fabricate advanced products that can meet today's demand. With all the advantages of 3D printing (complex design, low cost, and customized performance, etc.), multiple materials can provide additional flexibility for desired mechanical performance. Such a mechanical behaviour is highly desirable for example in 3D printing of tailor-made implants or soft robotics due to multiple requirements of varying stiffness and shorter production times [9, 10]. Multi-material FFF printing can be achieved using two or more extruders where nozzle temperature, printing speed, and resolution can be individually controlled as necessary. Back in 2010 due to the expiry of major FFF patents, FFF has expanded a lot in terms of reducing the cost of FFF printers and increasing design innovation in FFF printers. However even with so much advancement in FFF technology, there are still some issues that are halting the progress of FFF and these challenges need to be addressed. One of the major issues is low interfacial bonding strength in multi-material structure. Specifically, low interfacial bond strength is a significant issue in the FFF printing of dissimilar materials. Overall, this makes FFF AM technology a key manufacturing process for the fabrication of complex multi-material parts with less material waste.

\subsection{Objective}

For MM-AM to expand and grow from the current prototyping stage there are certain challenges that need to be addressed to fabricate multi-material parts. Currently, printing of discrete multi-material objects is a technically difficult process but an economically favourable manufacturing method, and it provides additional functionality and flexibility [11]. In the case of multi-material FFF (Fused Filament Fabrication), the interfacial bond at the joint between different materials is developed based on the fusion of one material into other materials, and the strength of the bond is highly dependent on the compatibility between two materials. This lack of fusion is the main reason for parts breakage at the interface of materials. Considerable research and analytical studies have been conducted to analyze the effect of interface on the overall strength of the 


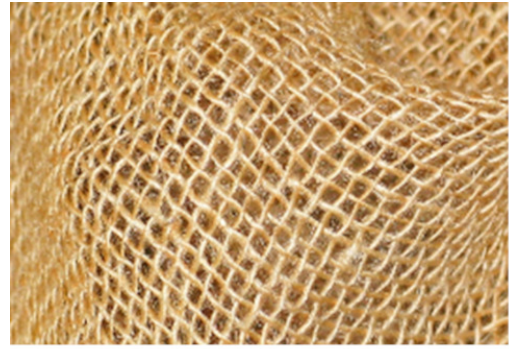

(a) Jute fibers

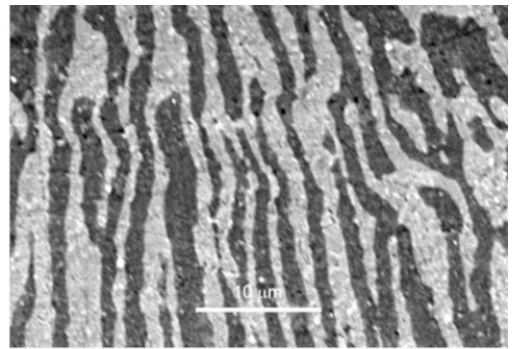

(b) Bi-Sn lamellae

FIGURE 1.2: Examples of human and nature intertwining materials: (a) Jute fibers are interlaced to form a tenacious cloth. (b) The lamellar microstructures of a Bi-Sn system are composed of alternating layers of bismuth (white) and tin (black) @2003 by Charles and Cockburn (CC BY-NC-SA 2.0 UK).

part. To improve the strength of the interfacial bond, different interlocking joints were developed and analyzed [12]. This shows that a macroscopic interface interlocking or material fusion can improve the strength of the interfacial bond. Different strategies have also been implemented like an application of chemicals and redistribution of materials during post-processing to soften the material. However, these strategies still have the joint weaker than the materials themselves. It is observed that humans often intertwine materials to strengthen a product, e.g., manufacturing of composites [13] and jute fibers (Fig. 1.23). Interlacing fibers in clothing fabric has multiple advantages including enhancing the durability and the resilience of a fabric [14]. Nature does the same too, like the lamellar microstructures in eutectic alloys (Fig.1.2p). Many AM processes, e.g., FFF, are like building and placing fibers, and the deposition way of these manufacturing technologies has a direct effect on the mechanical properties of the fabricated part. Based on these observations on how nature and humans enhance mechanical properties, we hypothesize that overlapping and interlacing materials at the interface will increase the bonding strength in MMAM. To test this hypothesis, one way is to expand the interface of different materials to a small overlapping region and generate the infill within the region with both the materials. Although overlapping various parts can be designed in the CAD phase, the generation of the interlaced infill and the toolpath needs to be done in the slicers. The capabilities of currently existing slicers are very limited in terms of the processing of material information in a multi-material model. In the case of a multi-material model with overlapping geometries, as shown in Fig. 1.3, these 


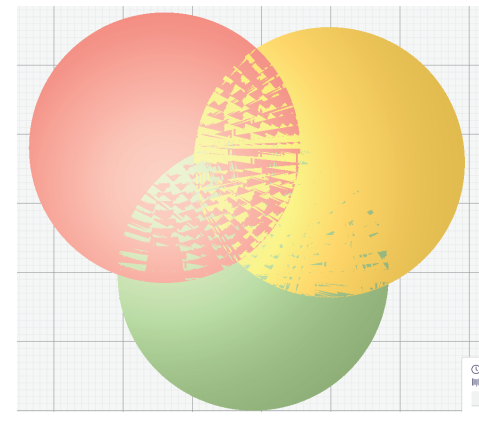

a

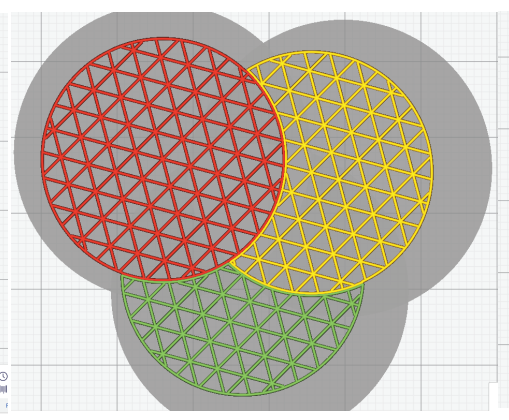

b

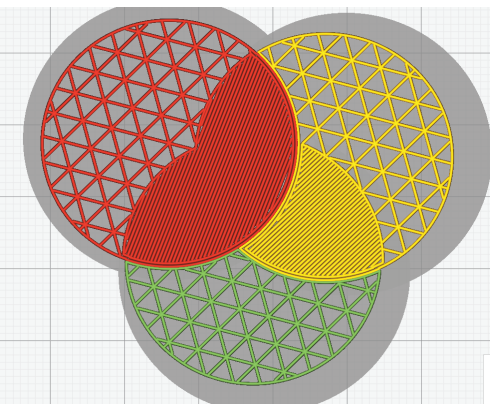

C

FIGURE 1.3: (a) A model having three overlapping cylinders (top view) is inputted in a commercial slicer - Ultimaker Cura. It either (b) lets one infill take over the overlapping region or (c) makes the region solid.

commercially available slicers randomly assign materials to the overlapping geometry. In this research we generated toolpaths as the result based on the FFF technology, but since the LDI has been applied to different technologies as well [15], the methodology can also be extended for other MMAM processes. This technology gap shows that the current computer-aided manufacturing (CAM) tools cannot make full use of material properties for MMAM. As such, we have to develop an advanced computational tool to process multi-material models. A ray representation - layered-depth images (LDI) [16] - is employed here, since it is a compact format containing volumetric information, and thus it can perform many tasks quickly such as Boolean operations and resolving mesh errors [17]. Making use of the materials specified in the CAD model, we develop a new format named layered-depth material images (LDMI) to encode the material information in the LDI sample-points. The sample-points encoded with material information can tell whether a region of a layer has a single material or multiple materials. Interlaced infills can then be generated for the multi-material regions.

\subsection{Major Contributions}

The major contributions of this research are summarized as follows:

1. A new computational tool is developed for MMAM, which can take a multimaterial CAD model as an input and use the material information in the slicing. 
2. Knowing the material distribution in different regions, infill interlacing is realized in the multi-material regions through toolpath planning.

3. Tensile test results show that interlaced infills increase the bond strength and outperform the T-joint interlocking. The fracture even happens outside the interface area, meaning that the bonding is at least as strong as the materials.

\subsection{Outline of the thesis}

The relevant reported studies on developments discussed in Chapter 2 so as to build essential background on this research topic. The development methodology for LDMI and technical concepts are introduced in Chapter 3. The experimental results are presented in Chapter 4. The major conclusions of the study are finally summarized in Chapter 5 together with the recommendations and desired future work. 


\section{Literature Review}

This research involves the computer-aided design and manufacturing of multi-material components with a focus on the enhancement of bonding between materials, so a brief review is done related to these fields.

\subsection{Multi-material additive manufacturing}

Different AM technologies are being used for MMAM [18] with each having its own advantages. Every additive manufacturing technology has some necessary common steps included in process planning like CAD design development, slicing and printing. For example, Polyjet uses the ink-jetting technology to jet different materials [19], selective laser melting (SLM) melts different powders together [20], digital light processing (DLP) changes materials by swapping the vats [21]. Among all these technologies, the most popular and easily accessed one is probably the FFF method [22, 23, 24]. This is because it is clean and can add more materials by using more extruders. MMAM technologies can be used to improve part performance by varying material compositions, which are not achievable by conventional manufacturing processes, and this opens multiple opportunities for design, functionality, and cost-effective high-value products [25, 26], especially in medical and dental [27] where high performance is required with desirable properties for biomedical applications. For example, two or more materials are printed using multiple extrusion nozzles in multi-material FFF polyjet, in which multiple STL bodies defined by discrete materials serve as the CAD input. In some other methods, multiple materials are used in such a way that one material acts as a support structure and can be easily removed once the print has been completed. Some examples of such 


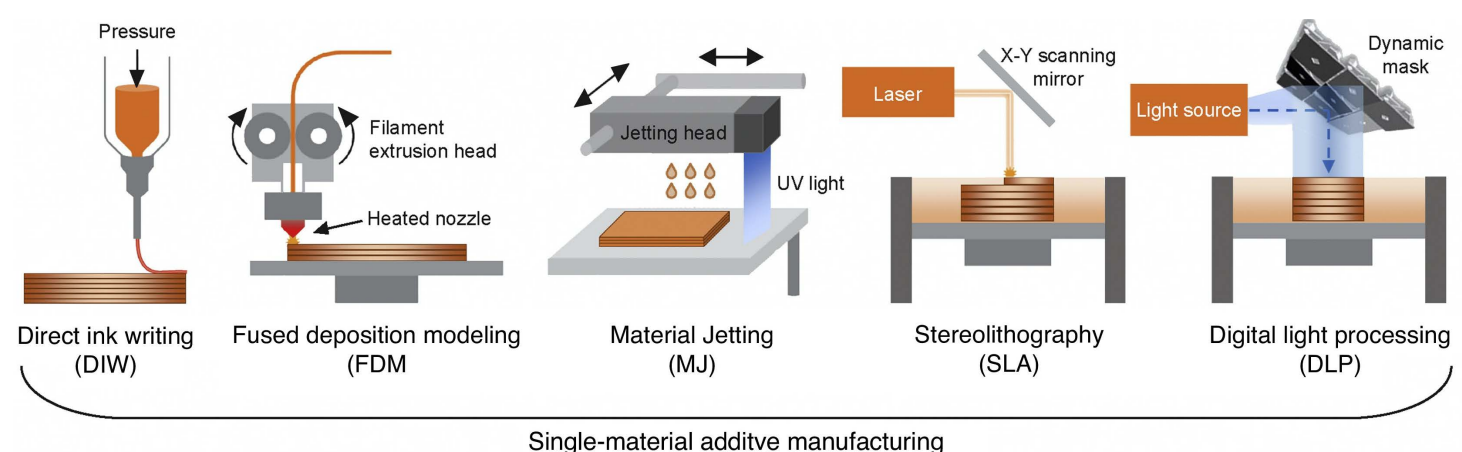

Single-material additve manufacturing

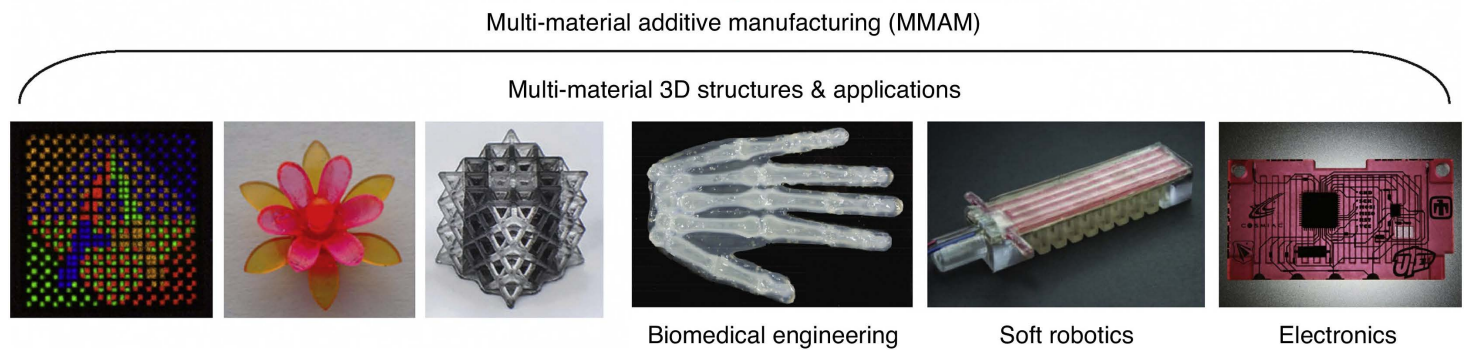

FIGURE 2.1: Additive manufacturing technologies and applications of multi-material additive manufacturing [41] @2020 by Elsevier.

work are presented in [22, 23]. Additive manufacturing has been recently used in different industrial application particularly for the fabrication of the multi-material structures like orthopaedic implants [28, 29]. Using the high precision and ultimate flexibility in geometric design make it possible for additive manufacturing to develop multi-material parts with customized mechanical properties [30, 31]. Due to the availability of various materials, there is a lot of innovation happening in MMAM but even though a lot of research has been done in the area of development of hardware for MMAM, there is a lot of work that needs to be done on the software side to make MMAM a viable commercial process [32]. Some application areas for MMAM includes soft robotics [33, 34], electronics [23, 35], 4D printing [36, 37], automotive [38, 39], aerospace [40]. Some application multi-material additive manufacturing is shown in Fig. 2.2 Despite much research has been done in this area, there are still many challenges, e.g., bonding between dissimilar materials, overall low interfacial bonding, and multi-material data processing from CAD to CAM [18, 41]. 


\subsection{Process planning for multi-material printing}

Researchers are contributing significantly to the development of modelling and designing strategies for MMAM using various spatial subdivisions schemes such as voxels [42. 43] and surfels [44]. For example, there is a digital material design framework proposed to find a proper material distribution by separating the whole domain into blocks and filling them up based on the tensor values [45]. However, there are some issues inherent in the current AM that has halted the progress towards MMAM [46]. Even the most advanced CAM tools for AM are based on conventional geometries and processes in AM [47]. Even with the multiple advances in FDM, there are still numerous critical barriers that restrict the enormous utility of MMAM. Multi-material printing has the potential to actually enable the vast industrial adoption of AM, but it suffers from the inefficient AM slicers that are based on conventional geometrical representations suited to old AM processes. Current MMAM slicers cannot utilize all the material information available in the CAD to the full extent [18]. In the past, multiple approaches have been tried to overcome the constraints in handling multi-material products. One of the approaches used to represent the multi-material model is a multi-CAD system [48]. This multi-CAD system is a set of STL models from available CAD modellers with solid information replaced by the material index. It requires users to specify the material information and the printing properties for each STL model separately, which adds certain complications to MMAM. A promising CAM tool is the LDI [49], which is based on ray-tracing. Compared to other slicing technologies like contour slicing and voxelization, ray-tracing is computationally efficient for detecting small features in complex AM structures [50]. Other than this, the related geometrical computations can be accelerated by modern graphics hardware equipped with a Graphics Processing Unit (GPU) by processing the pixels in parallel. Compared to the LDI, other existing methods may fail [51] or give poor performance [52] when the layer-complexity of an input model is high. Applying the LDI technique can potentially alleviate challenges in multi-material AM to manufacture functional products. 


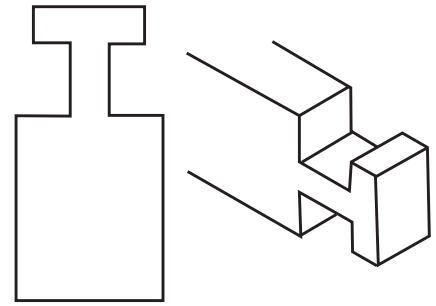

a

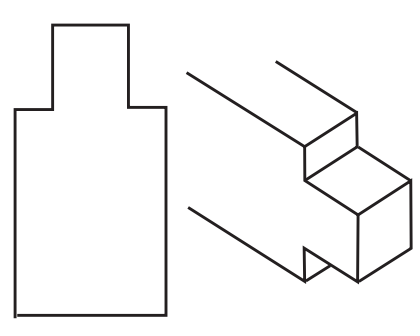

b
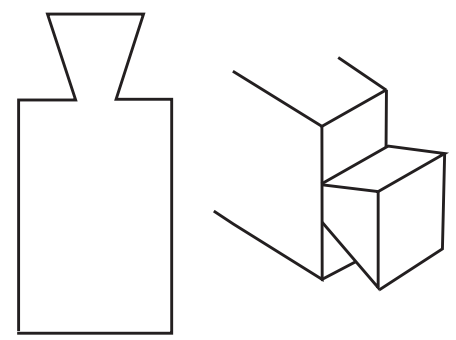

c

FIGURE 2.2: Types of Interface analyzed by Ribeiro et al. [12]. (a) T-shape (b) U-shape (c) Dovetail shape

\subsection{Interface and bonding between materials}

Ribeiro et al. [12] shown in Fig. 2.2 has analyzed the effect of different mechanical interlocking joints on the strength of the multi-material interface. They compared different materials and different interlocking joints (T-Shape, Dovetail Shape and U Shape) for analyzing the adhesion and interlocking strength. It was concluded that the strength of the interface joint is much more dependent on the macroscopic structure of the interface (mechanical interlock) than the material compatibility. In a similar research Garziosi et al. [53] conducted a detailed exploratory study on Fused Deposition Modeling (FDM) process for multi-material printing and confirmed that mechanical interlocking strategies results in increasing the adhesion and diffusion between different materials. Interface joints play a key role in the performance of a multi-material part and have been analyzed a lot in the past. Previously, various techniques have been tested to develop a strong interfacial bond between materials of different compatibility. Rossing et al. [54] presented a technique to control the bonding between flexible material like silicone and thermoplastics through mechanical interlocking. The bonding strength between silicone and thermoplastics was improved drastically using a hybrid fabrication process. Another research [55] analyzed the adhesive behaviour between materials in each layer and it was concluded that various printing parameters like the orientation of the print, printing direction and geometric design of the interface that directly affect the strength of the interface. Therefore, the strength at the interface can be improved by controlling these parameters. Lumpe et al. [56] also showed that strength at the material interface 
is directly related to the materials and the interface design. Some structures can even result in $20 \%$ increased strength using new multi-material designs. 


\subsection{Summary}

From the review of previous studies, it is evident that interfacial joints are the most critical areas in multi-material prints and the strength of the interfacial joint between two materials is dependent on the geometry of the interfacial joint. Moreover, design improvements in interfacial joints can enhance the mechanical performance of multimaterial structures. However, directly developing interlocking features like T-joints and lattice structures at the interface in the CAD model is a cumbersome and difficult process that requires a lot of time. 


\section{Methodology}

Suppose an object $H$ contains different material volumes, it can be defined as $H=O_{1} \cup$ $\cdots \cup O_{m}$. All these material volumes are defined by the separate material ID that helps in the identification of material present in that material region. There can be multiple single materials, double material or $n$ material regions. An overlapping material region is a material region that has two or more material IDs. For example an overlapping region between materials $A$ and $B$, for example, can be defined as $O_{A, B}=O_{A} \cap O_{B}$.

Here the goal is to identify the overlapping regions efficiently in the slicing process. By using these overlapping regions, this research generates an interlaced infill. This research purposes a new Layered Depth-Material Images (LDMI) technology developed on top of the Layered Depth Image (LDI) [49], which was developed based on the raytracing technology to sparsely encode a solid model by a set of intersection points between the rays and the model. As the name suggests, the main difference is the inclusion of material information in the sample-points. This material information present on the sample-points is very important for the detection of different material regions in the slicing process.The full processing pipeline of the proposed LDMI framework is shown in Fig. 3.1

LDMI slicing framework is developed on top of LDI technology which has been used in previous research to perform Boolean operations i.e. Union, Subtraction and Offsetting on the input mesh. The overlap between multi-material CAD geometries can be easily created in the LDMI slicing tool by performing the offsetting operations for each layer [57]. This allows us to modify mesh in the CAM phase of MMAM hence, there is no need to modify current multi-material CAD modelling approach and the multi-material models can be imported without any design modification. 


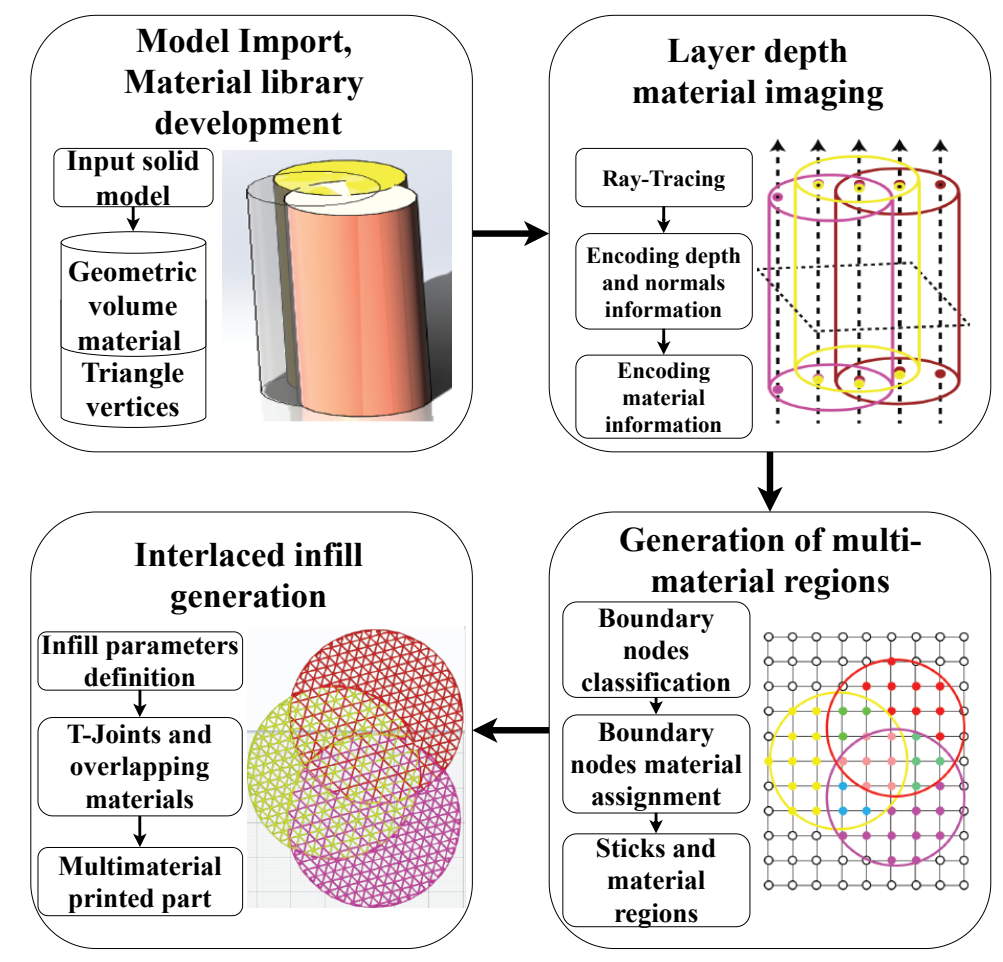

FIGURE 3.1: The workflow of the LDMI framework for processing and modeling multimaterial interface.

Firstly, given the input multi-material solid model $H$ (e.g., AMF file), the materials defined in $H$ are obtained and stored in a material library $(M)$. Each material $(m \in M)$ is given an index so that it can be retrieved at any stage of the process. Secondly, assume the build direction is aligned with the $z$-axis, a set of rays $(\gamma \in R)$ is shoot from the ground traveling along the build direction (i.e., $\widehat{\gamma}=(0,0,1))$ to intersect with $H$. The intersection points ( $p \in R \cap H$ ) are stored together with its material index correspondingly in each ray. Thirdly, slicing at a height is to place a two-dimensional (2D) plane parallel to the ground at the height to intersect with all the rays. Each grid node is corresponding exactly to one ray so that the materials of the node can be determined by its ray. Finally, the grid nodes are grouped into different regions according to their materials, and the interlaced infills are developed in the multi-material regions.

In summary, LDMI initially reads an input solid model $H$ as an AMF file and develops a material library that stores the material index for each polygonal face present in an 
input mesh. Then in step 2, LDMI develops $Z-L D M I$ images using ray-tracing technology and these LDMI images are then processed in step 3 to generate multi-material regions. In step 4, multi-material regions are utilized for developing interlaced infill and interlocking patterns in overlapping material regions to strengthen the interface between dissimilar materials. Lastly, tensile testing was utilized to assess the enhancement of mechanical properties due to interlaced infill in multi-material dob-bone samples.

In the developed multi-material slicing framework all the rendering programs, geometric computations, and slicing programs are accelerated by modern graphics hardware using NVIDIA CUDA kernel functions. This enables us to efficiently generate LDMI sample-points for complex input model $H$ in less time even when the layercomplexity of an input model is high. This is advantageous because other slicing techniques may fail [51] or give a poor performance at higher layer-complexity [52] of an input model. The technical details are presented in the following chapters, starting from the ray-tracing.

\subsection{Ray-tracing with material information}

An important process in the development of the LDMI program was to utilize the material information available in the input model $H$ to generate multi-material LDMI sample-points $(S)$. One of the most important steps in LDMI is the generation of sample points and details of the development of LDMI sample points are presented here. LDMI represents a solid model by an image which is basically a set of the array of pixels viewed from a single camera with parallel rays passing through the centers of pixels and intersecting with the model. Each intersection between the ray and a triangle of mesh generates a sample point. These rays come out from the image perpendicularly and all of them are parallel to each other.

Simply ray-tracing shoots a ray from the pixels to intersect the solid model obtaining a set of sample-points and each pixel stores necessary information about the intersection occurrence. For each sample point three values are stored which are; its coordinates, normal vectors, and material. The coordinates and the normal vectors give the geometry 


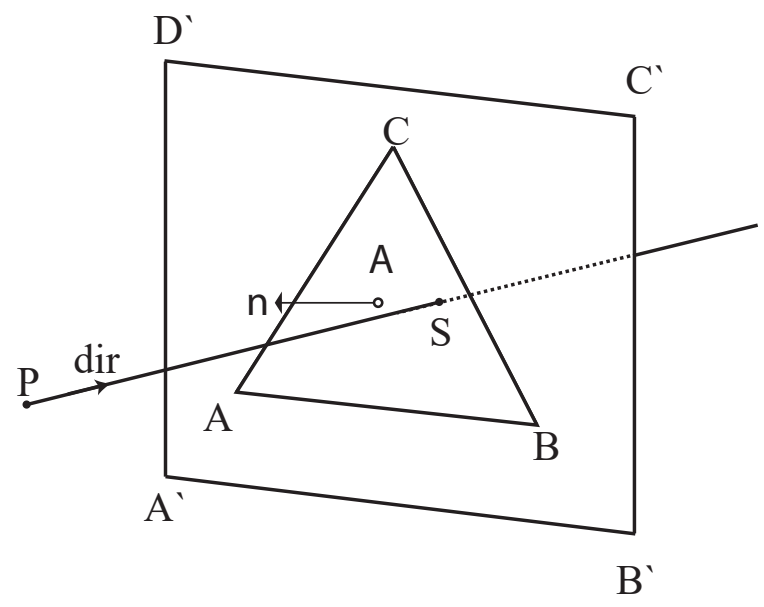

FIGURE 3.2: Ray tracing schematic diagram.

and the orientation information locally, and the material information are used to define the material regions. The image can be placed anywhere theoretically, but since the goal of slicing is to capture the changes in the cross-section geometry at different heights, the image is placed at the ground and the rays are shot in the build direction. The image should be large enough to cover the whole model, and the image resolution is set by the resolution of the $3 \mathrm{D}$ printer.

LDMI representation is dependent on the material information specified in the $H$ so using AMF file format LDMI takes polygonal mesh as an input. The AMF file format is used because it is currently the most widely adopted file format for the processing of multi-material models. The material data in the AMF file is stored for each volume of the model defined by a set of triangular faces. The first step in the LDMI program is to read this input AMF file for material data and vertices (A, B and C) of each triangular face. Fig 3.2 shows ray-tracing procedure for a triangle with material $A$; a point $P$ ray with origin $O$ and direction dir intersects a triangle defined by the vertices, $A, B$, and $C$ at intersection point $S$. In the second step, this information is stored in a structured material library with the face index of the triangle. This material library containing material information and geometric information is used in the ray-tracing process. 


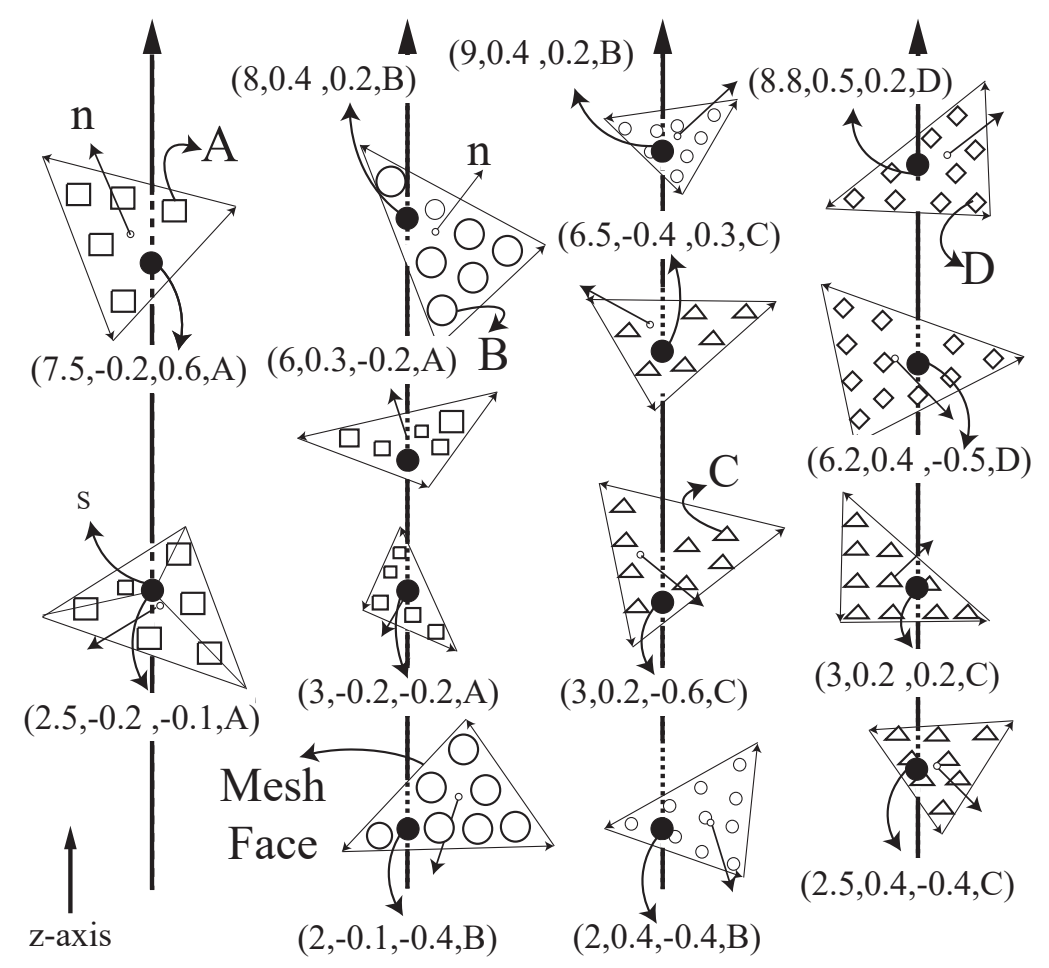

FIGURE 3.3: Ray tracing process to obtain the LDMI sample points.

An example of a ray with a single material $(A)$ is shown in Fig, 3.3 where LDMI generates entering and exiting sample-points $S$ for ray intersection with mat - $A$ triangles. Similarly rays in Fig 3.3 have material status of $(B, C$ and $D)$. The sample points can be obtained by computing many line-triangle intersections in the mentioned raytracing process as illustrated in Fig. 3.3, but it is very time consuming and inefficient. Therefore, a similar rendering technique [49] presented in LDI is applied here, and it is extended to include the material information.

The main difference between the LDMI and LDI technology is the recording of material information at each intersection point of a ray. In LDMI technique, each samplepoint stores material $m$, depth $d$ and normals $n_{x}$ and $n_{y}$ information. Material information is very important for sampling solids for detection of different material regions in the $H$ and for developing interlaced infill and interlocking joints at a multi-material interfacial bond. Applying the LDI technique here can potentially solve one of the biggest challenges in multi-material AM to manufacture objects with multiple materials. 
Rendering a model in the 3D space is to project and convert its triangles into a raster image located on the screen. Rasterization is usually used for rendering 3D models and it is basically a process of converting a vector graphics format (Triangles) into a raster image which is basically a series of pixels. Rasterization actually "projects" triangles onto the screen and this means that a model is transformed from 3D space to $2 \mathrm{D}$ representation using perspective projection. This is accomplished by projecting the vertices of triangles of an input mesh onto the screen. In comparison to ray-tracing which is very complex process, rasterization can only be computed in just two steps and this makes rasterization a very efficient process. In the developed LDMI program, all the geometrical computations are accelerated by modern graphics hardware equipped with a Graphics Processing Unit (GPU) by processing the pixels in parallel.

In engineering applications, the 3D solid models are represented in polygonal form using usually triangular mesh and there are three conditions for triangle rasterization.

1. A mesh is not a non-manifold mesh and rasterization does not leave any holes which means there are no non-rasterized pixels.

2. No triangle is rasterized more than once.

3. A center of a pixel in rasterization lies completely inside the triangle

Using these conditions rasterization fills up the image pixels based on the shapes of the triangles as well as their properties like colors. In other words, if a triangle is rastered on a pixel, it must have an intersection with the ray from that pixel. The color values for each pixel can be used to return the required information. The color value for each pixel was assigned during shading.

$R G B A$ color can be defined by four floating points: $(r, g, b, a)$, for the red, green, blue, and alpha channels. They are used to store the point's depth value $(d)$, two components of the normal vector $\left(n_{y}, n_{z}\right)$, and material index $(m)$. The depth value $d$ is the distance from the image, which is sufficient to retrieve the actual coordinates because the ray equation is known, and the third component of the normal vector can be calculated by the other two: $n_{x}=1-n_{y}-n_{z}$. Simply, a LDMI sample point is represented as 
$\left(d, n_{y}, n_{z}, m\right)$, and it is stored in the ray of the corresponding pixel where it is located. Fig. 3.3 shows an example of sample points and the values are represented in the format which are represented as $\left(d, m, n_{x}, n_{y}\right)$,

With the help of a graphics processing unit (GPU), hardware acceleration can be done to achieve real-time rendering to rasterize a 3D model and a single GPU renderer can outperform 20 CPUs due to the number of processors. This shows how in comparison to ray-tracing rasterization is extremely fast and therefore used in this research. For example, in this research: the NVIDIA GeForce 920M, generates a $1024 \times 1024$ LDMI for an input mesh of 282,624 triangular faces that takes only $294 \mathrm{~ms}$.

\subsection{Slicing for multiple materials}

After processing material information available in the input CAD model and after converting the CAD model to the LDMI representation, the LDMI solid is used in the slicing process, which divides the model as a stack of 2D layers. LDMI representation is a set of different sample-points and using these sample points it develops multi-material boundaries $(C)$ for every material present in $H$. By intersecting a slice plane with the rays of LDMI, a 2D grid is formed at a particular height. It is important to mention here that the normal for slicing plane and the LDMI rays both same direction of Z-axis. To obtain all the geometry and material information in each 2D slice, there are two tasks:

1. Determining the materials for each grid node

2. Forming different material regions.

LDMI framework is based on ray tracing in $z^{\prime}$ direction, hence, there are no limitations of minimum layer height, as LDMI program can generate infinite number of the slicing planes between the maximum and minimum $z$-coordinates of sample-points in LDMI solid. This provides LDMI an advantage to achieve fine quality prints. In this research a dual extruder Ultimaker - 3 Fused Filament Fabrication (FFF) 3D printer was used with the layer height of $0.18 \mathrm{~mm}$ for fast and good quality prints. 


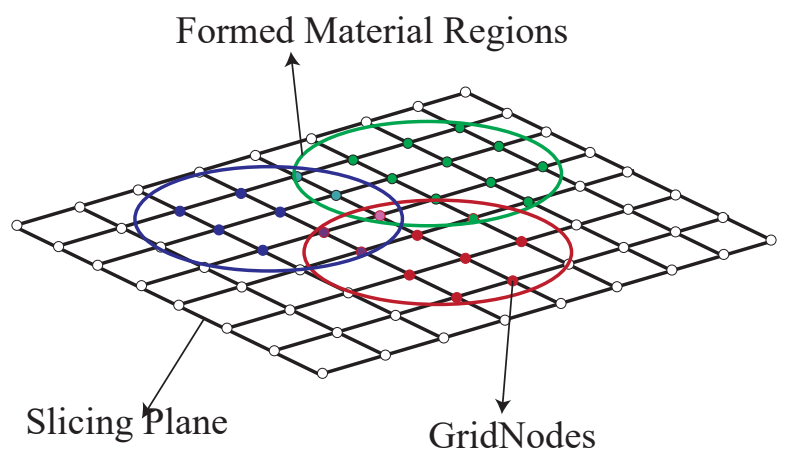

FIGURE 3.4: Slicing plane with classified gridnodes and material regions.

As shown in Fig: 3.4, a slicing plane is simply a set of gridnodes with different material assigned and these gridnodes are used to form multi-material regions. Depending on the material of LDMI samples points and the height of the plane, LDMI program determines whether a gridnode is inside or outside the material region. Only the gridnodes inside the LDMI solid are classified with material status are used to develop material regions which are then used to generate interlaced infill and interlocking pattern.

Now the next step in LDMI program is development of multi-material regions and details are presented here.

\section{Determining materials for grid nodes}

For every slicing plane, a pool of gridnodes is generated with specified resolution as shown in Fig: 3.4. and this section explains the approach for determining material status for grid nodes. Since the grid nodes are located in the rays, the sample points of the rays can be used to determine whether a grid node has any material and what materials it has. The method is illustrated in Fig. 3.5, where three rays having different sample points are shown. Notice that the $z$-axis is pointing to the right instead of the top in this figure, so virtually the grid nodes aligned vertically are from the same slice plane. The sample points (patterned circles) are located arbitrarily in the ray because their positions depend on the shape of the model, but the grid nodes (dots) are uniformly distributed 

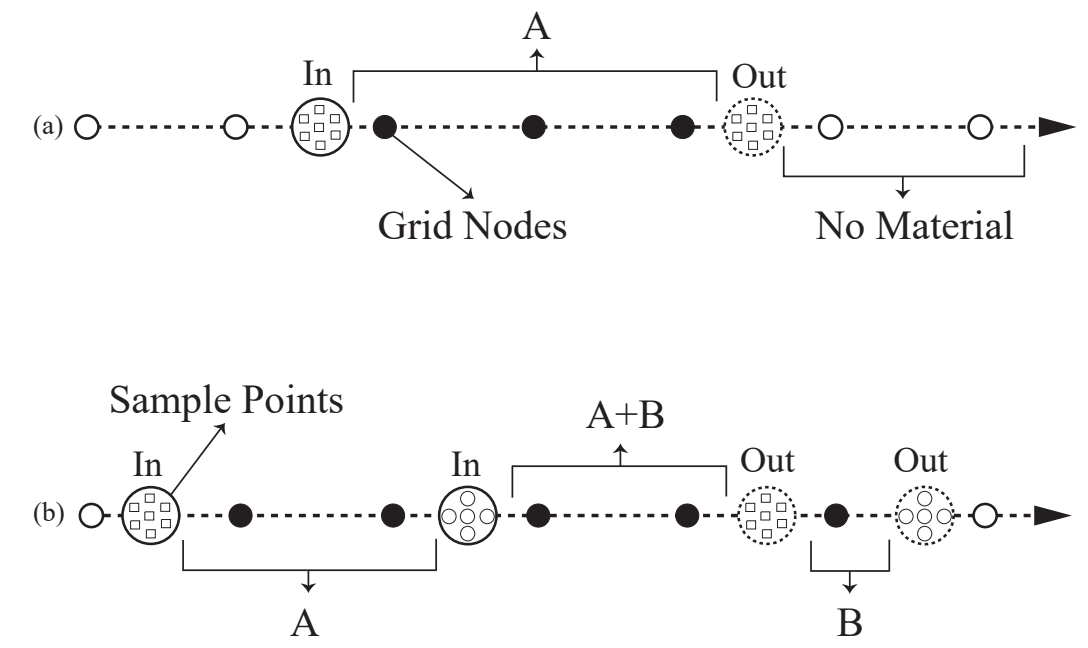

(c)

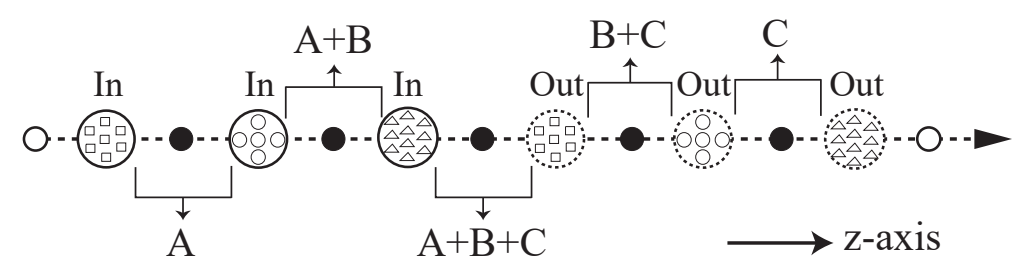

FIGURE 3.5: Determining materials for grid-nodes by LDMI sample points. Note that the $z$-axis is pointing to the right.

along the ray since all the layers have the same height. The solid-line and the dottedline circles indicates the In/Out of the sample points, where In means that the ray $(\gamma)$ is going into the model at the sample point, and Out means coming out. This In/Out classification can be done easily by the normal vector $\mathbf{n}=\left(n_{x}, n_{y}, n_{z}\right)$ of the sample point. It is an In point if $\mathbf{n} \cdot \gamma<0$, and it is Out if $\mathbf{n} \cdot \gamma>0$. For the case $\mathbf{n} \cdot \gamma=0$, the ray is a tangent line at the point on the model surface, and it is neither In nor Out, so it is ignored.

Starting sample-points for any material are represented with solid boundary patterned circles and all the gridnodes that are lying between the starting and ending sample-points are shown with a solid circle. Given the In/Out classification and the materials of the sample points, the materials of the grid nodes can be determined based on where they are located among the sample points. For example, the first two grid nodes (hollow dots) in Fig. $3.5 \mathrm{a}$ are located before the In-point, so they are not inside 
the model and should have no material. Similarly, the last two grid nodes also have no material as they are after the Out-point. Only the nodes (solid dots) located in between the In- and Out-points have materials. This can be extended to multiple materials too: if a node is located in between the In- and Out-points of material $A$ as well as the Inand Out-points of material $B$, then it contains both materials $A$ and $B$, and so on (see Fig. $3.5 p$ \& c).

For assignment of material index, LDMI program scans all the In gridnodes and assigns material values to each gridnode using the material values stored for samplepoints during the ray-tracing process. As shown in Fig. 3.5(b) and Fig. 3.5(c) Various active materials between the starting and ending sample-points on a ray. To store the material index of each gridnode LDMI program utilizes the power of hash-maps and store material information in a key-value pair with 'gridnode index' as a key and 'materials list' as a value. 'Materials list' is the list of all the materials that are active for a particular gridnode. This mapped data structure enables the LDMI program to efficiently scan for the materials index in the process of development of material boundaries for every material region.

With the help of the LDMI sample points, the material assignment can be done efficiently, and it has a constant computational complexity: $O(k)$, where $k$ is the number of sample points in a ray which is usually small up to a few tens depending on the shape complexity of the model. It is also worthwhile to mention that, unlike other slicing algorithms, the LDMI does not have to be updated even the number or the height of layers are changed. No matter how many grid nodes are used or how they are redistributed, the sample points are unaffected. This provides LDMI with an advantage to achieve fine quality prints without extra computational costs. LDMI gridnodes classification algorithm uses CUDA atomic operations to classify gridnodes. LDMI program uses gridnodes as an input and loop over all the possible gridnodes on a slicing plane. For any gridnode lying on a slicing plane inside the LDMI solid should have $z^{\prime}$ position coordinate located in between the depth of staring sample-point and ending samplepoint of a material. Fig. 3.5 shows the starting sample-point of a material represented 
by a solid circular boundary, contrarily, the ending sample-point for the same material is represented by a dashed circular boundary.

\section{Forming material regions}

After all the above, the grid nodes of a slice plane are assigned with materials, the 2D grid essentially is a discrete material field. The next step is to extract clear boundaries to bound different material regions so that the fabrication can be planned differently in each region. The boundaries are used in the interface modelling and the development of interlaced infill in the multi-material regions. Classified gridnodes are arranged in form of vertices of cubic cells on the slicing plane as shown in Fig. 3.6. LDMI slicing program sequentially scans all these cells using the marching cubes technique to identify boundary cells which are then used to develop boundaries of different material regions. Here, the marching squares algorithm is applied to the generation of contours for the 2D material field. Specifically, the algorithm processes four neighbour nodes at a time forming a cell and determines contour lines needed to represent the part of the contours that pass through this cell. The individual lines are then fused into the desired contours. The contour lines are constructed based on the cell configuration, i.e., the material combination. Some configurations and the output lines for the cells are shown in Fig. 3.6. In short, a line is placed between the nodes having different materials. If all the nodes in a cell have no material or the same material, no lines are generated for the cell. For a line that is placed between a node with no material and a node with a material (Fig. 3.6(a)), it is a part of the external boundary, i.e., the model surface. For a line that is placed between different materials (Fig. 3.6 (b-d), it is a part of the internal boundary. These are the ones that will be used to develop the interlocking patterns. After all the cells are processed and the contours are completed, a linear interpolation is applied along the contours to make them smooth. All the lines that are generated for each material region are arranged in a specific direction to form a closed contour for each material region. These material regions are utilized in the next function of LDMI program for the development of interlocking patterns and intertwined infill the multi-material regions. 


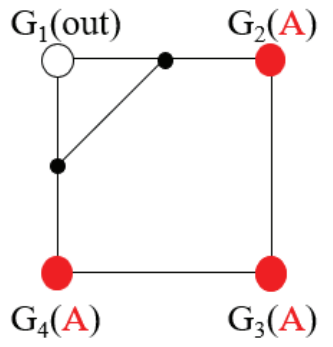

(b)

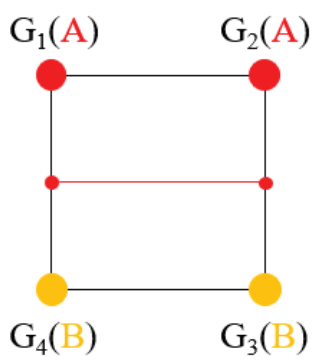

(c)

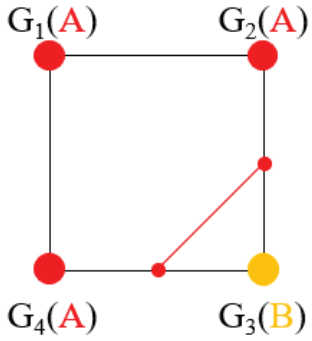

(a)

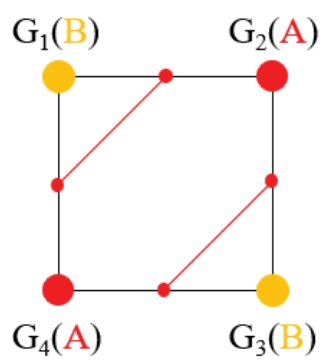

(d)

FIGURE 3.6: Development of contour lines in cells with ' $G$ ' representing grid-nodes on a slicing plane.

\subsection{Modeling multi-material interfaces}

Interfacial joints in the multi-material parts are the most critical stress areas that affect the mechanical properties of a multi-material structure. The LDMI framework utilizes previously formed multi-material regions to develop interlocking pattern (T-joint) and interlaced infill to enhance mechanical strength at these interfacial joints. Although the interlocking patterns are not the focus of this paper, the details of generating the T-joints are also presented here to demonstrate the generality of this framework. This developed LDMI technology can generate the T-joints on-the-fly in the CAM phase and this method does not require any cumbersome editing in the design process. Given the material interfaces and regions defined in a $2 \mathrm{D}$ plane, the details for generation of the interlaced infill and interlocking pattern are presented.

\section{Interlaced infills}

The material regions generated from the LDMI solid for any input model $H$ can be classified and can have a single material or multiple materials. Generation of infill is dependent on the material information of each material region. Interlacing infill in 
LDMI program is only dependent on the material regions developed in the previous step and does not require any design modification in the input solid model $H$. A normal infill is used for a single-material region, and an interlaced infill is generated for a multi-material region. It is important to mention here that tool-path development is an independent process for all the material regions, and this gives much more flexibility in developing tool-path according to the desired mechanical properties for each material regions. This means that LDMI slicing program is efficient to vary the infill generation properties for each material region as desired. Without loss of generality, triangular infills are used here to explain the generation of interlaced infills, but other types of infills can also be applied similarly.

To further enhance the interlacing effect, the LDMI program generates an infill to print alternate material on top of each other for every layer. Specifically, in overlapping region, the infill lines are interlaced between layers as well by printing them in alternate ways such as: Layer-1 (MA at bottom, and MB at top) and Layer-2 (MA at top, MB at bottom).

A triangular infill has three sets of parallel lines inclined at angles of $0^{\circ}, 60^{\circ}$, and $120^{\circ}$. The lines are placed uniformly separated by a distance $\Delta$, which is determined by the desired infill ratio $I$, i.e., the larger the infill ratio, the smaller the line distance. To develop an interlaced infill in the multi-material regions, the overall infill ratio $I$ is divided by the number of materials in the region. For example, if a region has $n$ materials, the infill ratio for each material is $I_{m}=I / n$, and the line distance is $\Delta_{m}=n \Delta$. The infill lines of different materials should be arranged in a stagger, so an infill shift (s) is also introduced, which is based on the line distance but applied separately to each material, i.e., $s_{1, \ldots, n}=\{0, \Delta, \ldots,(n-1) \Delta\}$.

In this section of research details of the generation of the interlaced infill in multimaterial regions is presented. First step in the generation of multi-material intertwined infill is the specification of infill properties for every material forming the multi-material region. Because multi-material regions are made of more than one material, due to this reason infill of a multi-material region is evenly divided into infill for each material that 


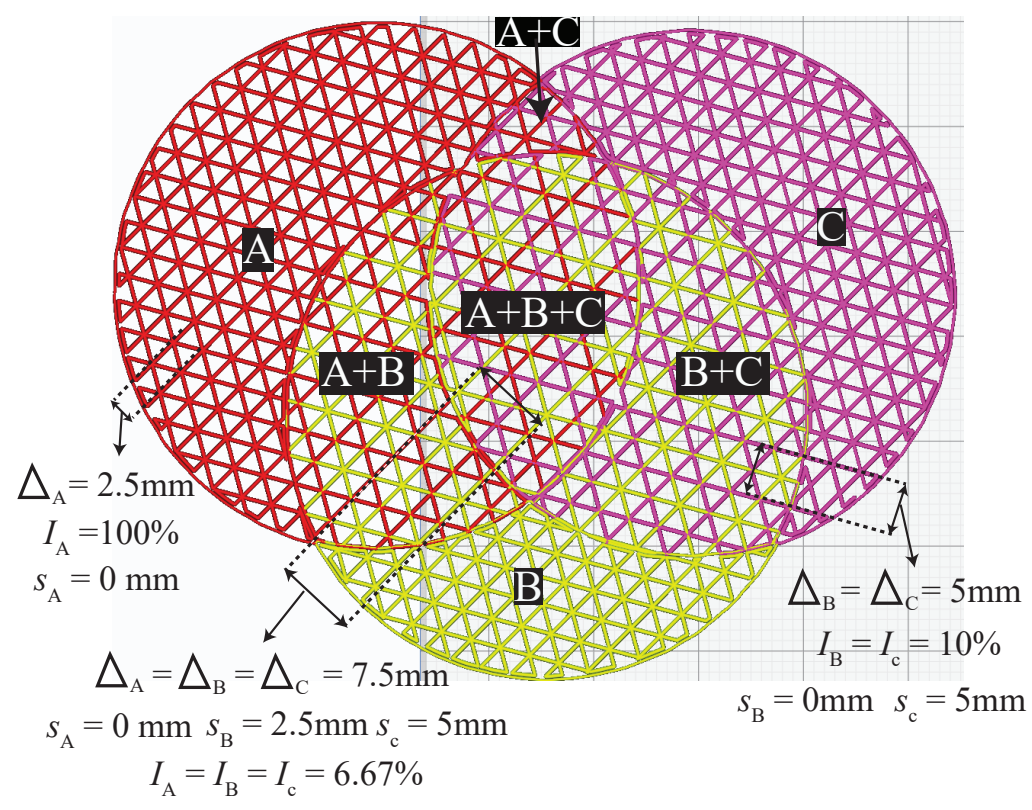

FIGURE 3.7: Visualization of interlaced infills on a model of three overlapping cylinders with materials $A, B$, and $C$.

makes up the multi-material region. Using the infill ratio $I$ of whole material region, infill ratio for each material $\left(I_{A}, I_{B}, I_{C}\right)$ can be calculated using this simple equation

$$
I_{A}=1 /(\text { total materials }) \times I
$$

These infill ratios are used to calculate the 'Infill Line Distance' as shown in Fig: 3.7 'Infill Shift' is another important parameter that is used to shift infill lines of a material relative to another material forming the multi-material region.

An example of three overlapping cylinders with materials $A, B$, and $C$ is shown in Fig. 3.7. There are seven different material regions, including three single-material regions, three double-material regions $(A+B, B+C, A+C)$, and one triple-material region $(A+B+C)$. An important property is the overall infill ratio $I$ that specifies overall infill of a material region and it can be in range of $0 \%-100 \%$. In the example the overall infill ratio is $I=20 \%$ and thus the line distance is $\Delta=2.5 \mathrm{~mm}$. In the single-material regions, the infill shift $s=0$ as there is no need to shift the infill lines. In the double-material regions (e.g., $A+B$ ), the whole infill is constituted by the two materials. Each of them occupies half of the infill volume, and thus the infill ratio for 


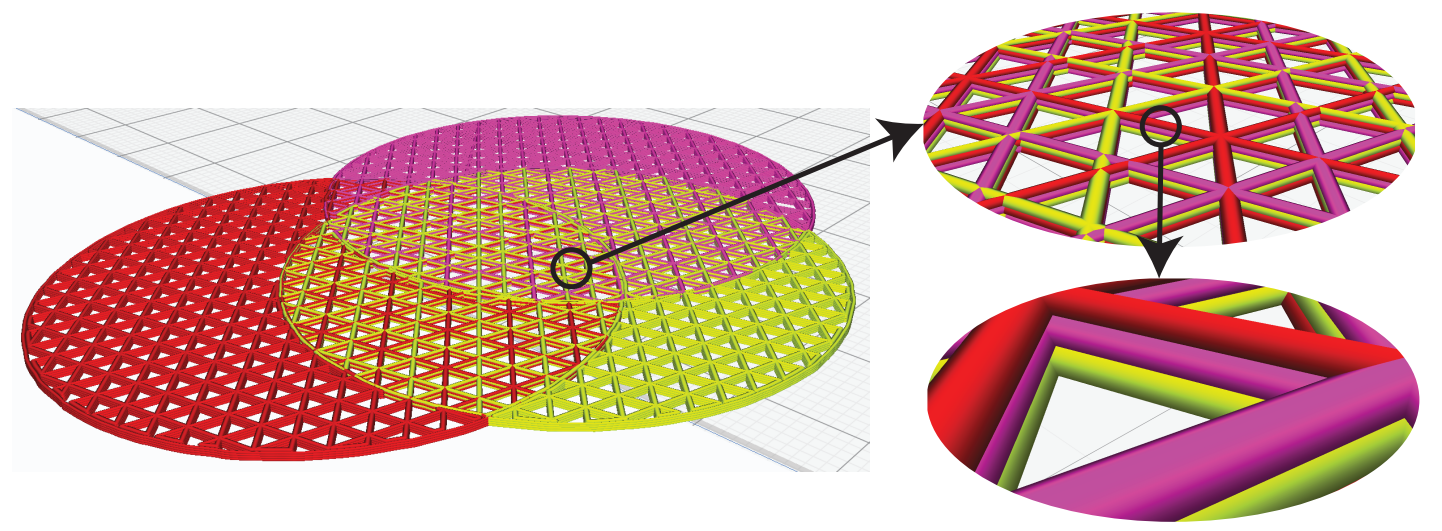

Isometric View

Magnified views

FIGURE 3.8: Infill shift parameter applied to materials $A, B$, and $C$ alternatively in subsequent layers to enhance interlacing effect.

the materials are $I_{A}=I_{B}=10 \%$ leading to their line distances $\Delta_{A}=\Delta_{B}=5 \mathrm{~mm}$. To alternate the materials, one material is shifted by the line distance $\Delta$, i.e., $s_{A}=0 \mathrm{~mm}$ and $s_{B}=2.5 \mathrm{~mm}$. Similarly, in the triple-material region $(A+B+C), I_{A}=I_{B}=I_{C}=6.67 \%$, $\Delta_{A}=\Delta_{B}=\Delta_{C}=7.5 \mathrm{~mm}, s_{A}=0 \mathrm{~mm}, s_{B}=2.5 \mathrm{~mm}$, and $s_{C}=5 \mathrm{~mm}$. As shown in example Fig. 3.7 infill lines of different materials are extruded on top of each other and edges of infill triangles are printed with different materials. To further enhance the interlacing effect among layers, the infill shifting is applied to the materials alternatively in subsequent layers. For example, after printing layer 1 with the above values, layer 2 uses $s_{A}=2.5 \mathrm{~mm}, s_{B}=5 \mathrm{~mm}$, and $s_{C}=0 \mathrm{~mm}$, so that the order is $A \rightarrow B \rightarrow C$ in layer 1 , then $C \rightarrow A \rightarrow B$ in layer 2 , and so on. This can interlace the materials not only in $2 \mathrm{D}$ but also uniformly throughout the whole model and this is shown in Fig. 3.8 .

\section{T-joint}

Ribeiro et al. [12] found that the T-joint is the best interlocking pattern to improve the mechanical performance at the interface of two materials. Therefore, it is used to compare with the proposed interlacing infill. Instead of adding the joints explicitly in the CAD model, the framework is general enough to directly create these patterns at the 
material interface during the toolpath planning, and the method is presented here.

Recall that the contours are generated in Section 3.2 for the external (model surface) and internal boundaries (between material regions). The internal boundaries are the material interfaces, and they can be altered to form T-shapes. To develop new T-joint sticks, the LDMI algorithm takes number of required T-joints as an input to define the dimensional properties of a T-joint. Specifically, a T-shape as shown in Fig. 3.9 can be defined by its widths at the top $\left(W_{t}\right)$ and the bottom $\left(W_{b}\right)$ as well as its heights for the top-half $\left(H_{t}\right)$ and the bottom-half $\left(H_{b}\right)$. One T-shape can be drawn by seven line segments $\left(l_{1}, l_{2}, \ldots, l_{7}\right)$, where $l_{1}=l_{7}=H_{b}, l_{2}=l_{6}=W_{t}-W_{b}, l_{3}=l_{5}=H_{t}$, and $l_{4}=W_{t}$. These seven segments are stored as a subprogram and programmed in incremental coordinates (e.g., G91 in G-Code), so that the subprogram can be called directly to generate the T-joints at the desired locations.

The T-joints are created at the time of toolpath generation for the internal boundaries. When a part of the internal boundaries is at least $W_{t}$, the original segment of length $W_{b}$ is removed and replaced by the subprogram of the T-shape, based on the orientation of the original segment. After one T-joint is generated, the tool travels a distance of $W_{h}$ before the next T-joint. In this way, a T-joint is also made in the opposite side. This process is done for both materials sharing the boundary, and the result is illustrated in Fig. 3.9. The offset for the line width is done using the tool radius compensation in G-code (i.e., G41 or G42). The amount of interlocking required at the interfacial joint is directly related to the T-Joints and by using desired value the desired percentage of interlocking can be varied within a range. Its important to mention here that a T-joint is saved as a preset for a model, and whenever a T-joint is needed, this preset can simply loaded by the user to replace an original sticks with T-joint. In this way, the inclusion of T-joints can be done simply in slicing stage rather than complex design modifications in CAD model. 


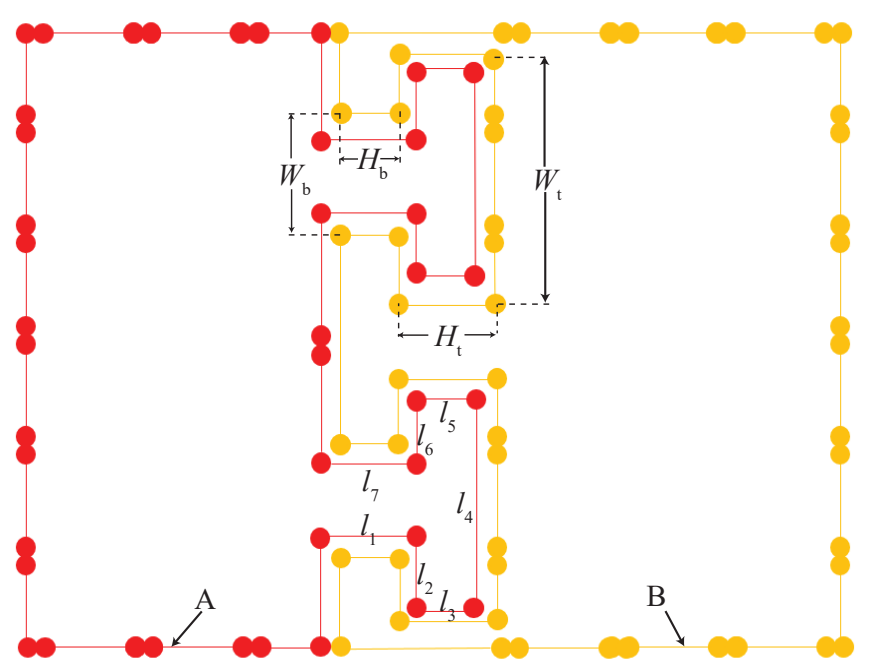

FIGURE 3.9: Development of T-joints at the interface.

\subsection{Summary}

This chapter of research presents details about the ray-tracing and the rasterization technology which are the main techniques used in the LDMI program to develop sample points with material information from the input solid model $H$. In the second part of this chapter details about the development and classification of grid-nodes with material information is presented. These grid nodes were utilized in the development of different material regions which were utilized in the development of different interlocking features (T-joint and interlaced infill) at the interfacial joint between different materials. The details for the generation of material regions are presented in last section of this chapter. 


\section{Results}

The proposed LDMI slicing framework was implemented as a $\mathrm{C}++$ program with Microsoft Visual Studio 2017. All the examples shown here are tested with Intel CORE i5 7th Generation with 8GM RAM and Nvidia GeForce 920M graphics card. The developed LDMI framework, can efficiently model the multi-material interface, and this section tests the initial hypothesis that the interlacing infill increases interface strength. This research is conducted on the hypothesis that interlocking features like T-joint and interlaced infill can enhance the mechanical properties in a multi-material structure. So to asses, the enhancement in mechanical properties due to different joint configurations and to measure the multi-material interface strength, samples with a dog-bone shape were fabricated with two materials touching at the middle of the dog-bone, as shown in Fig. 4.1. The overall infill ratio is set as $40 \%$, and the samples are 3D printed with an Ultimaker-3, which are made sure to have the same amount of material. These dogbone samples were analyzed using tensile test and stretched to their breaking point with a tensile tester - Mark-10 ESM750SLC (see Fig. 4.2), and the stress-stain data are obtained for the analysis.

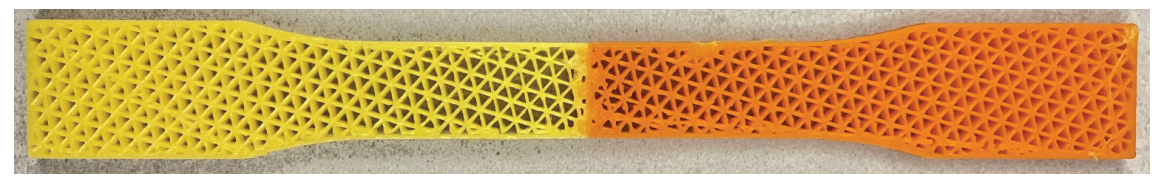

\section{T- Joint}

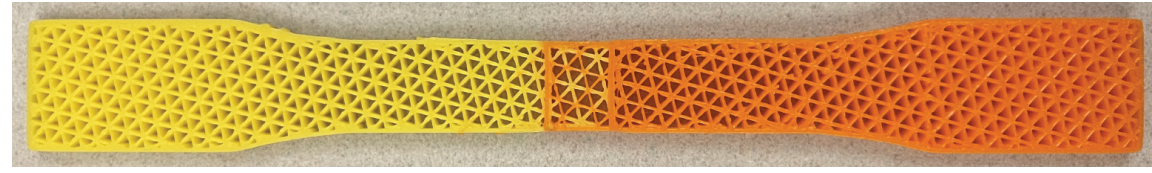

\section{$10 \mathrm{~mm}$ interlaced}

FIGURE 4.1: The dog-bone samples for tensile test. 


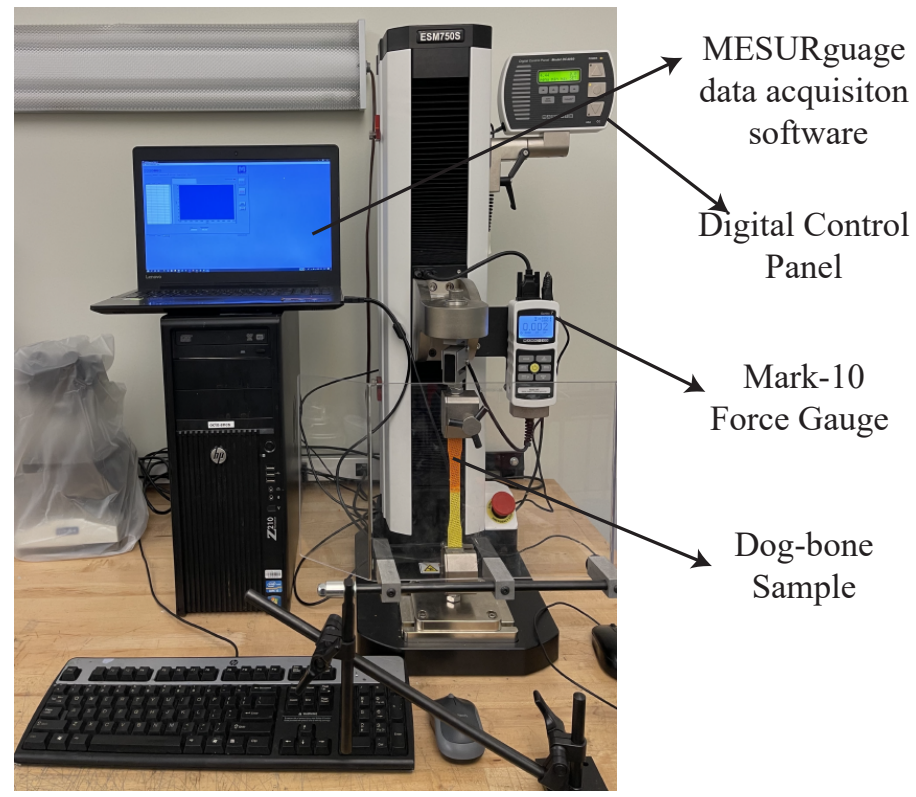

FIGURE 4.2: The experimental setup for tensile test.

Three sets of experiments are designed. For tensile test analysis, three sets of experiments were designed. For the first test case in this research, the same material (PLA) was used to print the dog-bone samples but with two different colors (orange and yellow). This allows us easily to compare the interface strength among various joint configurations. In the second experiment of this research, effectiveness of overlapping and interlacing was verified by analyzing the tensile tests of the different materials which are PLA, PETG and ASA. Lastly, in the end, results from the tensile tests were critically analyzed and a relationship was established between the interface strength and the overlapping amount of materials.

For all the different joint configurations of dog-bone samples, three samples were tested and all the tensile tests results were very similar. The results from all the configurations are averaged $( \pm 0.1 \mathrm{MPa})$. As a reference, all the materials used in the experiments are also tested individually with the same dog-bone shape, and their data are reported in Fig. 4.3, including the Young's modulus $(E)$ and the ultimate tensile stress (UTS). It can be seen that the PLA is much stronger and stiffer than the other two materials (PETG and ASA) and can carry at least two times more load before fracture. 


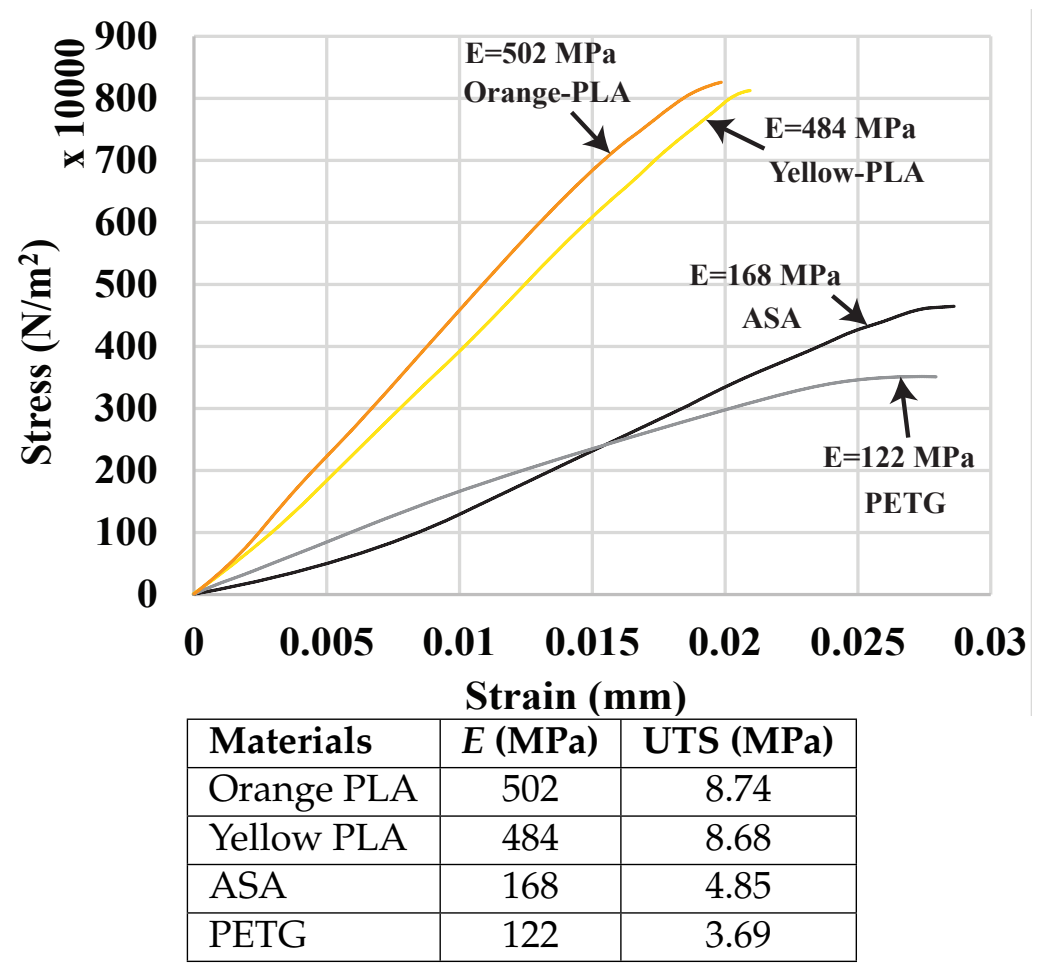

FIGURE 4.3: Tensile test data for single-material samples.

\subsection{Joint configurations}

The bonding strength of dog-bone samples with different interface and different joint configurations was compared and analyzed and the results are presented here:

1. Butt Joint.

2. Interlocking T-joint.

3. Proposed overlapping joint with interlaced infill

Butt joint was developed by placing the materials closely next to each other without any kind of design modification. To print dog-bone samples with butt joint a dog-bone sample was simple divided into two equal parts and a different material was assigned to each half part. The interface strength for this joint purely depends on the material compatibility and the adhesion between the material surfaces introduced in the 3D printing process. It is used as the baseline to show the improvement achieved by other configurations. The strategy for the development of the T-joints and interlaced infill joint 


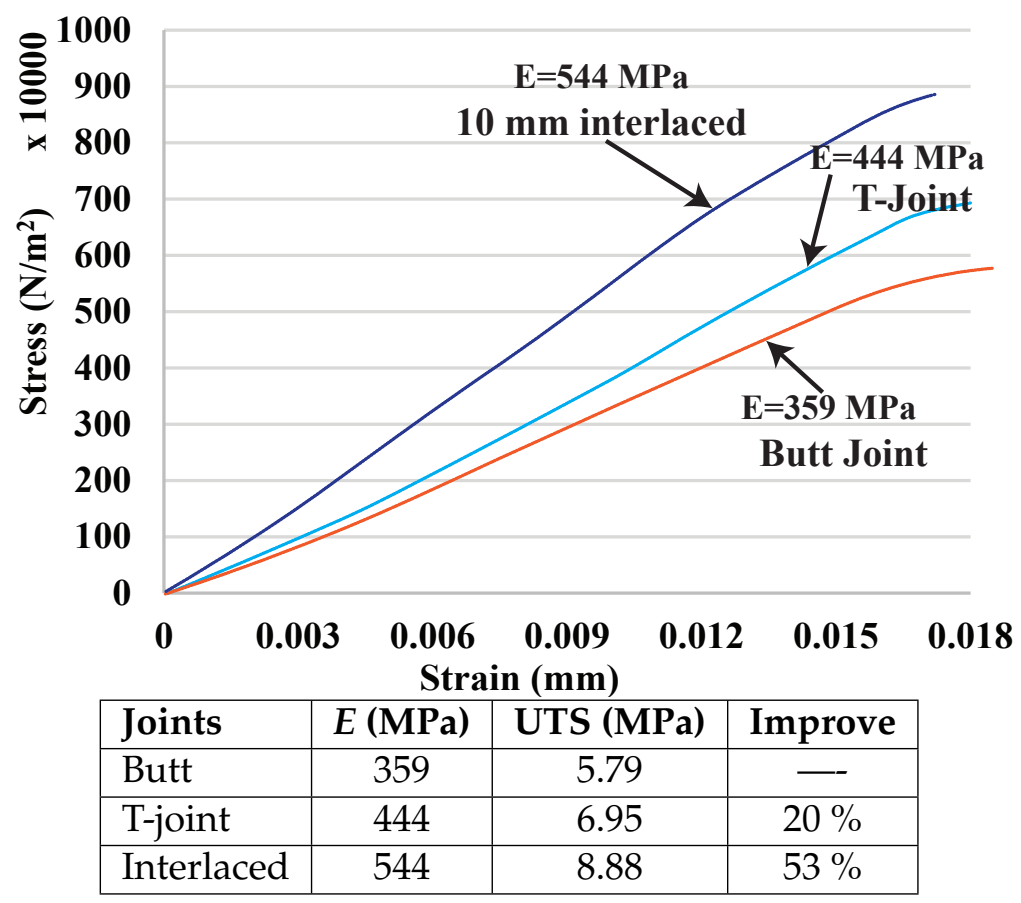

FIGURE 4.4: Tensile test data for different joint configurations.

configurations is explained in 3 In the tested dog-bone samples featuring T-joint configuration the T-joint has sizes of $W_{t}=2.10 \mathrm{~mm}, W_{b}=0.85 \mathrm{~mm}, H_{t}=1.35 \mathrm{~mm}$, and $H_{b}=0.62 \mathrm{~mm}$. In the tested dog-bone samples featuring interlaced infill, has an overlap size of $10 \mathrm{~mm}$. To make the effect of joints prominent, similar materials (orange PLA and yellow PLA) are tested in this experiment.

The testing results are shown in Fig. 4.4 and the fractured samples are shown in Fig. 4.5. For the butt joint configuration, the observed $E$ value is $359 \mathrm{MPa}$ and the observed UTS is $5.79 \mathrm{MPa}$, which are far less than the strength of the PLA materials themselves (see Fig. $4.3-E$ is around $500 \mathrm{MPa}$ and UTS is around $8.7 \mathrm{MPa}$ ). In case of butt joint the fractured samples shows that the fracture happens exactly at the joint interface. This reveals that the weakest location of a multi-material structure is indeed at the interface, and the amount of reduced strength is significant ( $\sim 40 \%$ reduction).

The T-joint in the samples, at the interface, introduces a mechanical interlock effect in addition to the adhesion. The tensile test results of the dog-bone samples are in line with the findings in the previous work [12] that the T-joint can improve the interface strength. These results show that T-joint achieved $20 \%$ increase in UTS compared to the butt joint 


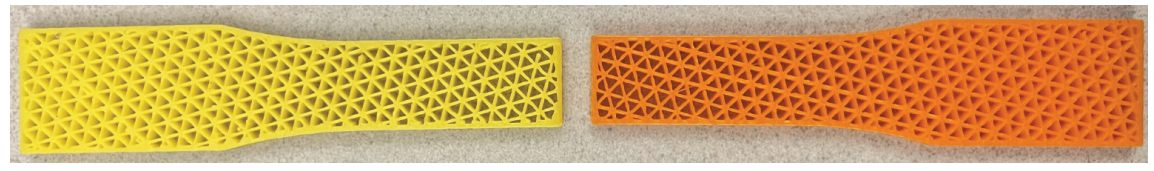

\section{Butt Joint}

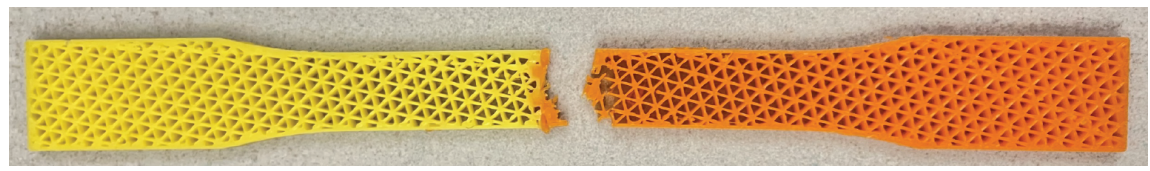

T- Joint

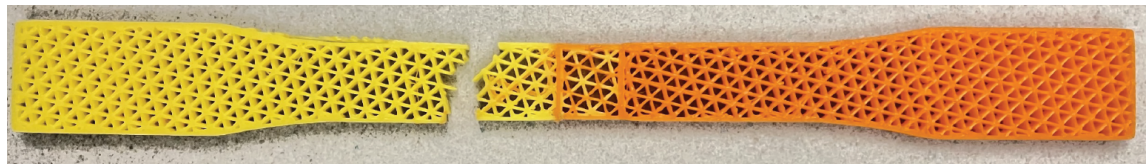

$10 \mathrm{~mm}$ interlaced

$20 \mathrm{~mm}$

FIGURE 4.5: Fractured samples of butt joint, T-joint, and interlaced infill.

(6.95 vs. $5.79 \mathrm{MPa}$ ). However, it is still $20 \%$ weaker than the PLA materials, and the fracture also happens at the joint. The proposed interlaced infill shows a very promising result. The sample actually fractured at a distance of $12 \mathrm{~mm}$ away from the joint area, meaning that the joint is at least as strong as the materials. This is evidenced by the observed $E=544 \mathrm{MPa}$ and UTS $=8.88 \mathrm{MPa}$, which is not only a $53 \%$ improvement compared with the butt joint, but also as strong as the PLA materials. The results prove that overlapping and interlacing materials can increase the interface strength between similar materials.

\subsection{Materials with distinct properties}

In this research practicality of the developed LDMI multi-material slicer is demonstrated by the printing of dog-bone samples with material combination of (PLA-ASA, PLA-PTEG). In the previous experiment, two similar materials (orange PLA- yellow PLA) were used, so in this experiment the interlaced infill was analyzed using the distinct materials to further confirm the effectiveness of interlaced infill. After analyzing the tensile test results of different interfacial joint configurations, the $10 \mathrm{~mm}$ interlaced infill joint configuration achieved the best mechanical performance. This shows that 


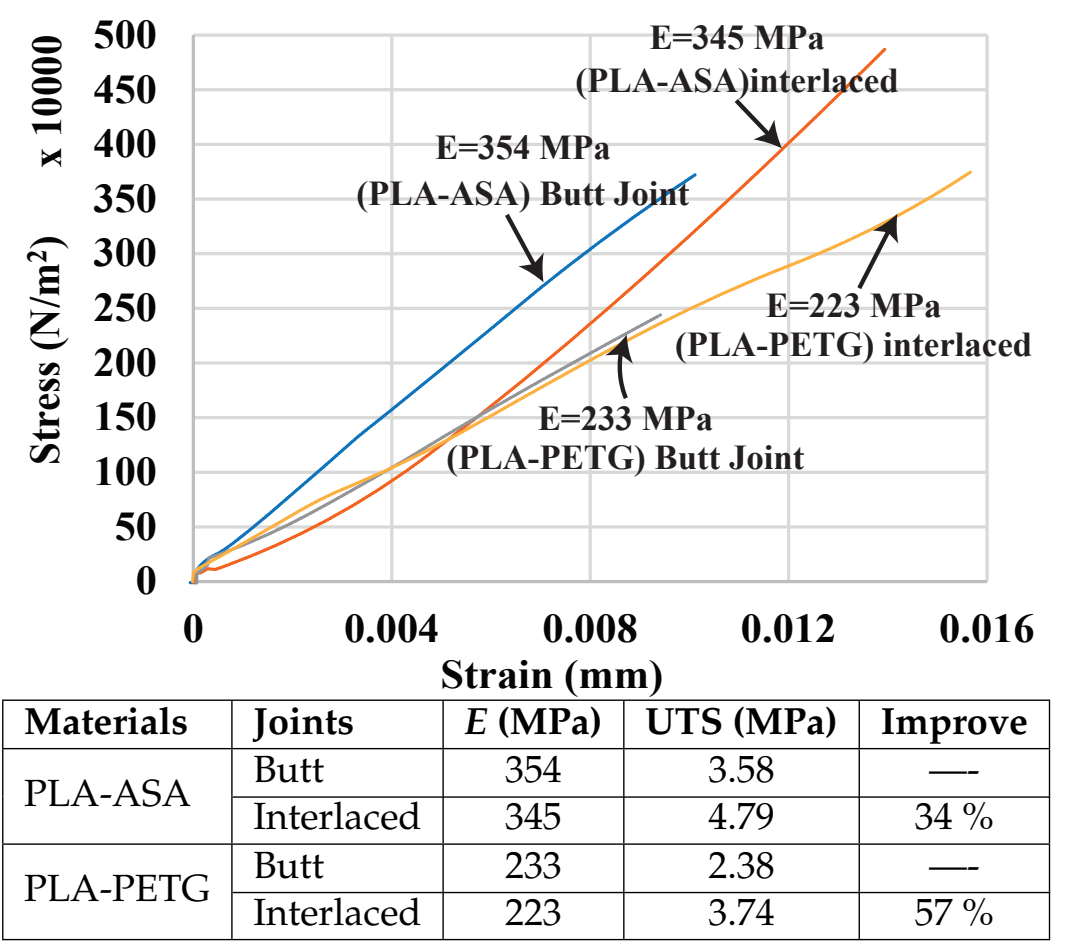

FIGURE 4.6: Tensile test data for different material combinations and joint configurations.

even for the material combination of multi-material dog-bone samples, $10 \mathrm{~mm}$ overlap will have the strongest interfacial bond.

Each material combination is tested with both the butt joint and the interlaced infill, and the overlap size for the interlaced infill is also $10 \mathrm{~mm}$ in this experiment. The testing results are shown in Fig. 4.6 and the fractured samples are shown in Fig. 4.7. Both material combinations show the same trend with the results in the experiment using similar materials. The tensile test results show that the interface strength of the butt joint is lower than the weaker material in the material combination and this happened due the material incompatibility between the dissimilar material forming the butt joint. For example, the UTS of the material ASA is $4.85 \mathrm{MPa}$, but the UTS of the combination PLA-ASA is only 3.58 MPa. Additionally, like the previous example the fractures of the butt joint always happen at the interface. In contrast, the samples with interlaced infill fractured inside the weak material at a distance away from the joint. They also have the UTS of the weak material, achieving an improvement of $34 \%$ and $57 \%$, respectively for PLA-ASA and PLA-PETG, compared with the butt joint. This reveals that the interface 


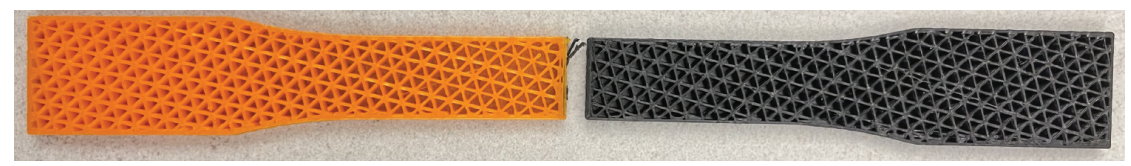

(PLA-ASA) Butt Joint

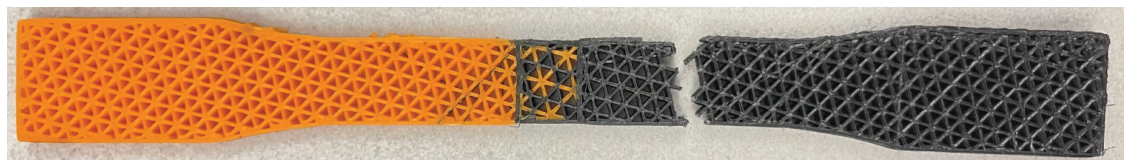

(PLA-ASA) 10 mm interlaced

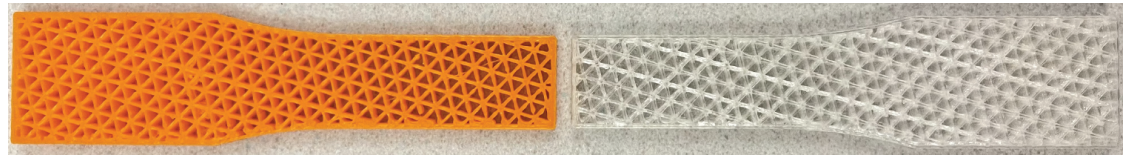

(PLA-PETG) Butt Joint

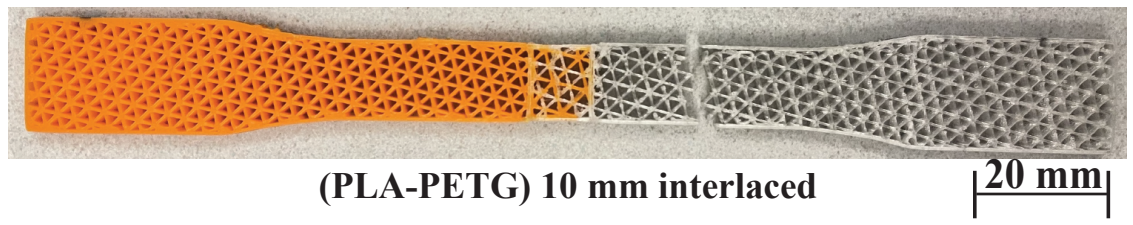

FIGURE 4.7: Fractured samples of butt joints and interlaced infills for the material combinations: PLA-ASA and PLA-PETG.

is even stronger than the weak material and it is because the interlace infill is developed with PLA material which is much stronger than the either ASA or PETG.

The results of these two experiments verify initial hypothesis that the interlaced infill can enhance the multi-material interface strength in 3D printing. The present method can also be potentially used for reinforcement by interlacing a stronger material with a weaker one.

\subsection{Overlap size and interface strength}

For detailed study of interlaced infill, dog-bone samples with different overlap/interlace size ( $5 \mathrm{~mm}, 10 \mathrm{~mm}, 15 \mathrm{~mm}, 20 \mathrm{~mm}$ ) were compared and a relation was developed between the mechanical properties and the size of the interlaced infill. To make the effect of interlace infill more prominent we printed the dog-bone samples with similar materials (orange PLA and yellow PLA) are tested in this experiment. After knowing that 


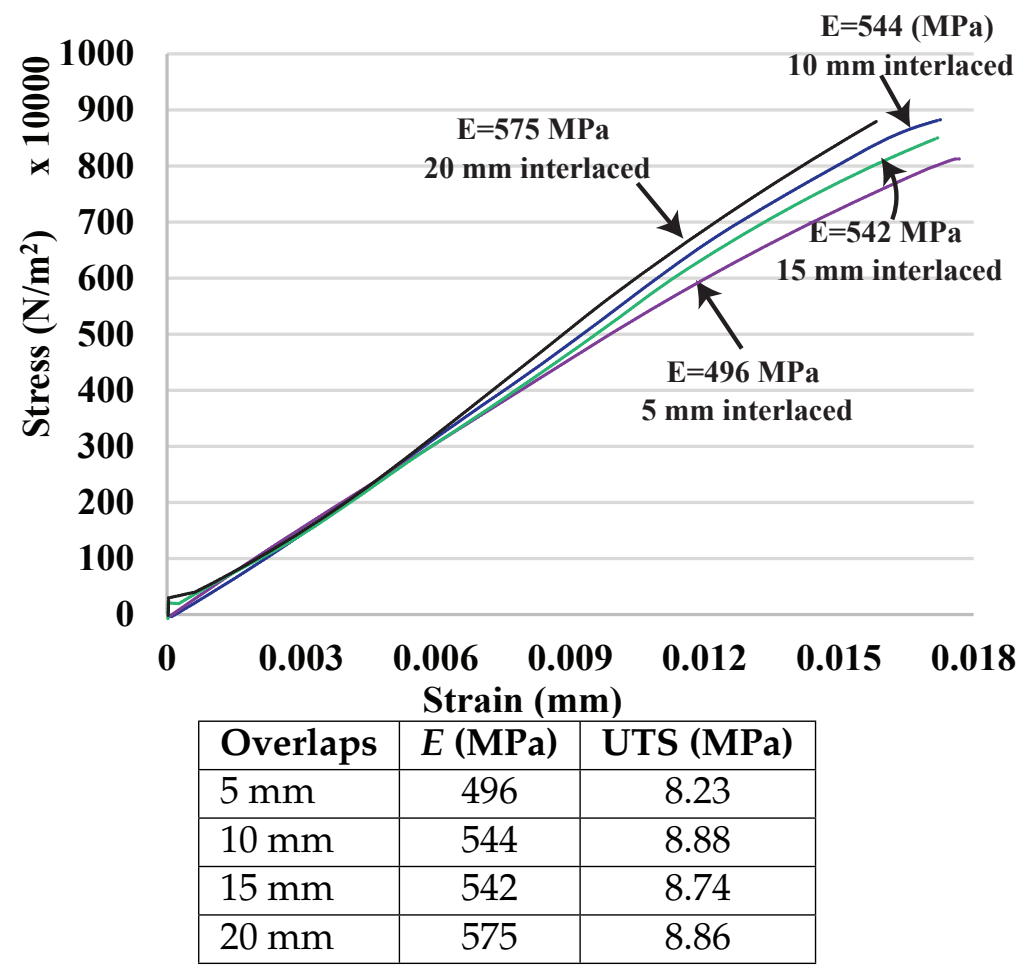

FIGURE 4.8: Tensile test data for different sizes of overlap.

interlacing infills is effective, this experiment wants to find out how critical the size of overlap is to the interface strength and what their relationship is.

The experiment is set up in the following way. First, the materials with similar property (orange PLA and yellow PLA) are used to better observe the changes due to the joint area. Second, four different sizes of overlap are tested: $5 \mathrm{~mm}, 10 \mathrm{~mm}, 15 \mathrm{~mm}$, and $20 \mathrm{~mm}$. The testing results are shown in Fig. 4.8 and the fractured samples are shown in Fig. 4.9 (the $10 \mathrm{~mm}$ one can be found in Fig. 4.5). From the data, it is observed that as the size of overlap increases from $5 \mathrm{~mm}$ to $10 \mathrm{~mm}$, the interface strength in terms of UTS is also higher from $8.25 \mathrm{MPa}$ to $8.88 \mathrm{MPa}$. This shows about $7.09 \%$ improvement in the value of UTS with $5 \mathrm{~mm}$ increase in size of interlace infill. It can also be seen that the sample of $5 \mathrm{~mm}$ overlap fractures in the joint area, so that the joint is indeed weaker than the PLA materials ( $\sim 8.7 \mathrm{MPa}$ ), but the difference is not large, and it is still better than the T-joint (6.95 MPa). By observing the fractured dog-bone samples it was clear that $5 \mathrm{~mm}$ interlaced infill size is not enough to create significant interlacing effect that can prevent fracture at the location of the interfacial joints. 


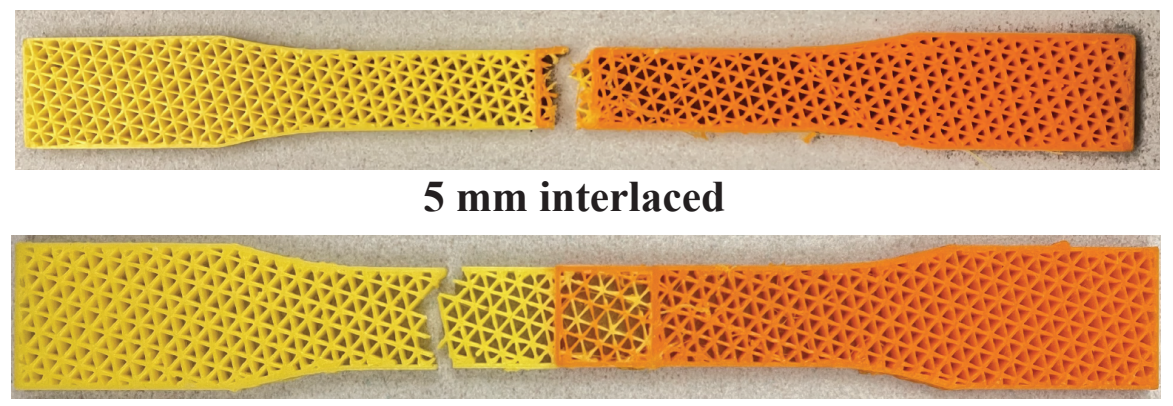

$15 \mathrm{~mm}$ interlaced

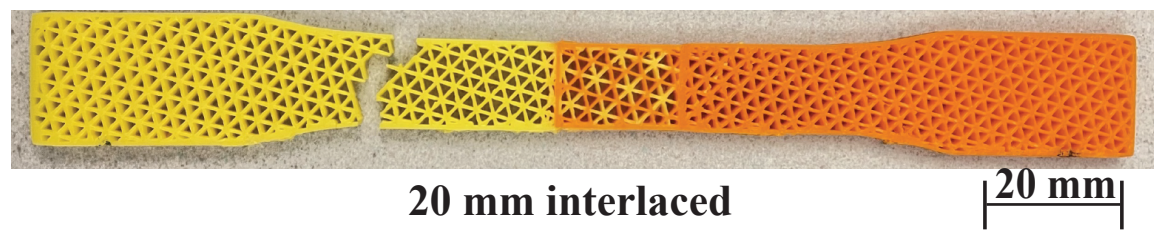

FIGURE 4.9: Fractured samples of interlaced infills with different overlaps: $5 \mathrm{~mm}, 15 \mathrm{~mm}$, and $20 \mathrm{~mm}$.

The $10 \mathrm{~mm}, 15 \mathrm{~mm}$, and $20 \mathrm{~mm}$ samples all fracture outside the joint, and they have similar UTS (8.77 - 8.88 MPa). All these samples fractured $(10 \mathrm{~mm}, 15 \mathrm{~mm}$ and $20 \mathrm{~mm})$ at the distance of about $12 \mathrm{~mm}$ from the boundary of interlaced infill; this shows that interlace infill size above $5 \mathrm{~mm}$ can effectively create an interlocking effect at interfacial joint that can prevent fracture at the location of the joint. The tensile test results of samples with $(10 \mathrm{~mm}, 15 \mathrm{~mm}$ and $20 \mathrm{~mm})$ interlaced infill reveals the performance is already maximized at the overlap size of $10 \mathrm{~mm}$, and the results are consistent beyond this size. Therefore, the overlap size can be set between 5 and $10 \mathrm{~mm}$ in practice, which can already provide a better property than mechanical interlocking joints. Overall after carefully analyzing the tensile tests for all different sizes of interlaced infill; it was concluded that in multi-material structures can achieve better mechanical properties by interlacing infill and for a certain size that can in multi-material structures can achieve best mechanical properties for a certain size of intertwined infill. 


\section{Conclusion and Future Work}

Based on the observation that materials in nature are often intertwined to increase in strength, this paper hypothesizes that overlapping and interlacing infills of different materials enhances the interface strength between the materials of a multi-material part. However, existing slicers do not have the capability to generate the interlaced infill. To test the hypothesis, this paper also develops a novel slicer for multi-material additive manufacturing (MMAM) to process the material information efficiently - named layered depth-material images (LDMI). The LDMI framework takes a multi-material computer-aided design (CAD) model as an input and applies rendering techniques to encode the material data into the sample points of the model. The encoded data are then used in the slicing process to identify different material regions in each slice plane, and the interlaced infills are generated in the multi-material regions. The framework can also generate the interlocking T-joints without any extra process in the CAD model.

Experiments are conducted to compare different joint configurations (butt joint, Tjoint, and interlaced infill) and different material combinations (PLA-PLA, PLA-ASA, and PLA-PETG). The tensile test results show that the T-joints are better than the butt joints, but the interlaced infills outperform both. The samples of interlaced infill even fracture at somewhere outside the joint area, meaning that the interlacing leads to a joint at least as strong as the materials. The trend is the same for different material combinations. This verifies the hypothesis, and the approach is promising to create strong multi-material parts. The method can also be applied to the reinforcement of a weaker material by interlacing it with a stronger material. It is also found that an overlap size of $5-10 \mathrm{~mm}$ is sufficient to have the full interlacing impact on the interface.

The major limitations of the present work include it is only tested with the triangular 
infills, the dog-bone shapes, and the fused filament fabrication (FFF) process. Triangular infill is normally the strongest infill pattern because triangles are least likely to deform. It will be interesting to find out whether the interlacing has the same impact on other infill patterns as well. We also plan to extend the present work to functionally graded infills by varying the infill ratio of each individual materials, which will require a new way to interlace the materials to achieve the desire mixing ratio. In addition, more complex shapes and loading conditions should be tested to further confirm the method's capability. Finally, it should also be tested on other MMAM process like MultiJet printing (MJP) to validate its generality. 


\section{Bibliography}

[1] Sunpreet Singh et al. "Current status and future directions of fused filament fabrication". In: Journal of Manufacturing Processes 55 (2020), pp. 288-306.

[2] Amit Bandyopadhyay and Susmita Bose. Additive manufacturing. CRC press, 2019.

[3] Ian Gibson et al. Additive manufacturing technologies. Vol. 17. Springer, 2014.

[4] Amit Bandyopadhyay and Bryan Heer. "Additive manufacturing of multi-material structures". In: Materials Science and Engineering: R: Reports 129 (2018), pp. 1-16.

[5] Praveen Sreeramagiri et al. "Design and development of a high-performance Nbased superalloy WSU 150 for additive manufacturing". In: Journal of Materials Science E Technology 47 (2020), pp. 20-28.

[6] Zhenzhen Quan et al. "Additive manufacturing of multi-directional preforms for composites: opportunities and challenges". In: Materials Today 18.9 (2015), pp. 503512.

[7] Taban Larimian and Tushar Borkar. "Additive manufacturing of in situ metal matrix composites". In: Additive Manufacturing of Emerging Materials. Springer, 2019, pp. 1-28.

[8] Sasan Dadbakhsh et al. "Selective laser melting to manufacture "in situ" metal matrix composites: a review". In: Advanced Engineering Materials 21.3 (2019), p. 1801244.

[9] Nicholas Meisel and Christopher Williams. "An investigation of key design for additive manufacturing constraints in multimaterial three-dimensional printing". In: Journal of Mechanical Design 137.11 (2015).

[10] Skylar Tibbits. "4D printing: multi-material shape change”. In: Architectural Design 84.1 (2014), pp. 116-121. 
[11] Konstantinos Salonitis et al. "Multifunctional materials used in automotive industry: A critical review". In: Engineering Against Fracture. Springer, 2009, pp. 59-70.

[12] Micaela Ribeiro, Olga Sousa Carneiro, and Alexandre Ferreira da Silva. "Interface geometries in 3D multi-material prints by fused filament fabrication". In: Rapid Prototyping Journal 25.1 (2019), pp. 38-46. DOI: 10.1108/RPJ-05-2017-0107.

[13] Suong Van Hoa, Minh Duc Hoang, and Jeff Simpson. "Manufacturing procedure to make flat thermoplastic composite laminates by automated fibre placement and their mechanical properties". In: Journal of Thermoplastic Composite Materials 30.12 (2017), pp. 1693-1712.

[14] Yasir Nawab, Syed Talha Ali Hamdani, and Khubab Shaker. Structural textile design: interlacing and interlooping. CRC Press, 2017.

[15] Huachao Mao et al. "Adaptive slicing based on efficient profile analysis". In: Computer-Aided Design 107 (2019), pp. 89-101.

[16] Pu Huang, Charlie C. L. Wang, and Yong Chen. "Algorithms for layered manufacturing in image space", Book Chapter, ASME Advances in Computers and Information in Engineering Research". In: ASME Advances in Computers and Information in Engineering Research (2014).

[17] Yong Chen and Charlie C. L. Wang. "Regulating complex geometries using layered depth-normal images for rapid prototyping and manufacturing". In: Rapid Prototyping Journal 19.4 (2013), pp. 253-268.

[18] Mohammad Vaezi et al. "Multiple material additive manufacturing-Part 1: a review". In: Virtual and Physical Prototyping 8.1 (2013), pp. 19-50.

[19] Pitchaya Sitthi-Amorn et al. "MultiFab: a machine vision assisted platform for multi-material 3D printing". In: ACM Transactions on Graphics (TOG) 34.4 (2015), pp. 1-11.

[20] Ali Gokhan Demir and Barbara Previtali. "Multi-material selective laser melting of Fe/Al-12Si components". In: Manufacturing Letters 11 (2017), pp. 8 -11. DOI: 10.1016/j.mfglet.2017.01.002 
[21] Christopher-Denny Matte et al. "Automated storage and active cleaning for multimaterial digital-light-processing printer". In: Rapid Prototyping Journal 25.5 (2019), pp. $864-874$. DOI: $10.1108 / \mathrm{RPJ}-08-2018-0211$.

[22] RE Brennan et al. "Fabrication of electroceramic components by layered manufacturing (LM)". In: Ferroelectrics 293.1 (2003), pp. 3-17.

[23] Devin J Roach et al. "The $m^{4}$ 3D printer: A multi-material multi-method additive manufacturing platform for future 3D printed structures". In: Additive Manufacturing 29 (2019), p. 100819.

[24] David Espalin et al. "Multi-material, multi-technology FDM: exploring build process variations". In: Rapid Prototyping Journal (2014).

[25] Dongping Deng, Tsz-Ho Kwok, and Yong Chen. "Four-Dimensional Printing: Design and Fabrication of Smooth Curved Surface Using Controlled Self-Folding". In: Journal of Mechanical Design 139.8 (June 2017). 081702. DOI: 10.1115/1.4036996.

[26] Rouhollah D Farahani, Martine Dubé, and Daniel Therriault. “Three-dimensional printing of multifunctional nanocomposites: manufacturing techniques and applications". In: Advanced materials 28.28 (2016), pp. 5794-5821.

[27] J Schweiger et al. "Histo-anatomic 3D printing of dental structures". In: British dental journal 221.9 (2016), pp. 555-560.

[28] Susmita Bose, Sahar Vahabzadeh, and Amit Bandyopadhyay. "Bone tissue engineering using 3D printing". In: Materials today 16.12 (2013), pp. 496-504.

[29] Gianni Campoli et al. "Mechanical properties of open-cell metallic biomaterials manufactured using additive manufacturing". In: Materials E Design 49 (2013), pp. 957-965.

[30] Furqan A Shah et al. "Long-term osseointegration of 3D printed CoCr constructs with an interconnected open-pore architecture prepared by electron beam melting". In: Acta biomaterialia 36 (2016), pp. 296-309.

[31] Amir A Zadpoor and Jos Malda. Additive manufacturing of biomaterials, tissues, and organs. 2017. 
[32] Wei Gao et al. "The status, challenges, and future of additive manufacturing in engineering". In: Computer-Aided Design 69 (2015), pp. 65-89.

[33] Erina Baynojir Joyee and Yayue Pan. "Multi-material additive manufacturing of functional soft robot". In: Procedia Manufacturing 34 (2019), pp. 566-573.

[34] Arijit Ghosh et al. "Stimuli-responsive soft untethered grippers for drug delivery and robotic surgery". In: Frontiers in Mechanical Engineering 3 (2017), p. 7.

[35] A Muguruza et al. "Development of a multi-material additive manufacturing process for electronic devices". In: Procedia Manufacturing 13 (2017), pp. 746-753.

[36] Xiao Kuang et al. "Advances in 4D printing: materials and applications". In: Advanced Functional Materials 29.2 (2019), p. 1805290.

[37] Farhang Momeni, Xun Liu, Jun Ni, et al. “A review of 4D printing”. In: Materials $\mathcal{E}$ design 122 (2017), pp. 42-79.

[38] Arash Afshar and Roy Wood. “Development of Weather-Resistant 3D Printed Structures by Multi-Material Additive Manufacturing". In: Journal of Composites Science 4.3 (2020), p. 94.

[39] Ryosuke Matsuzaki, Takuya Kanatani, and Akira Todoroki. "Multi-material additive manufacturing of polymers and metals using fused filament fabrication and electroforming”. In: Additive Manufacturing 29 (2019), p. 100812.

[40] Joel C Najmon, Sajjad Raeisi, and Andres Tovar. "Review of additive manufacturing technologies and applications in the aerospace industry". In: Additive manufacturing for the aerospace industry (2019), pp. 7-31.

[41] Daehoon Han and Howon Lee. "Recent advances in multi-material additive manufacturing: methods and applications". In: Current Opinion in Chemical Engineering 28 (2020), pp. 158-166.

[42] Adeyemi Oladapo Aremu et al. "A voxel-based method of constructing and skinning conformal and functionally graded lattice structures suitable for additive manufacturing". In: Additive Manufacturing 13 (2017), pp. 1-13. 
[43] Sambit Ghadai, Anushrut Jignasu, and Adarsh Krishnamurthy. "Direct 3D Printing of Multi-level Voxel Models". In: Additive Manufacturing (2021), p. 101929.

[44] Mark W Jones, J Andreas Baerentzen, and Milos Sramek. “3D distance fields: A survey of techniques and applications". In: IEEE Transactions on Visualization and Computer Graphics 12.4 (2006), pp. 581-599.

[45] Yuen-Shan Leung et al. “Digital Material Design Using Tensor-Based Error Diffusion for Additive Manufacturing". In: Computer-Aided Design 114 (2019), pp. 224 -235. DOI: $10.1016 / \mathrm{j} . \mathrm{cad} .2019 .05 .031$.

[46] William Oropallo and Les A Piegl. “Ten challenges in 3D printing”. In: Engineering with Computers 32.1 (2016), pp. 135-148.

[47] Pierre Muller, Jean-Yves Hascoet, and Pascal Mognol. "Toolpaths for additive manufacturing of functionally graded materials (FGM) parts". In: Rapid Prototyping Journal (2014).

[48] Xiuzhi Qu and Noshir A Langrana. "A System Approach in Extrusion-Based MultiMaterial CAD 313". In: International Solid Freeform Fabrication Symposium. 2001.

[49] Charlie CL Wang and Yong Chen. "Layered depth-normal images: A sparse implicit representation of solid models". In: arXiv preprint arXiv:1009.0794 (2010).

[50] Tsz-Ho Kwok. “Comparing Slicing Technologies for Digital Light Processing Printing". In: Journal of Computing and Information Science in Engineering 19.4 (2019).

[51] Bruno Heidelberger, Matthias Teschner, and Markus Gross. "Real-time volumetric intersections of deforming objects". In: Vision, modeling, and visualization 2003. AKA. 2003, pp. 461-468.

[52] Fang Liu et al. "Freepipe: a programmable parallel rendering architecture for efficient multi-fragment effects". In: Proceedings of the 2010 ACM SIGGRAPH symposium on Interactive 3D Graphics and Games. 2010, pp. 75-82. 
[53] Francesco Tamburrino, Serena Graziosi, and Monica Bordegoni. “The influence of slicing parameters on the multi-material adhesion mechanisms of FDM printed parts: An exploratory study". In: Virtual and Physical Prototyping 14.4 (2019), pp. 316332.

[54] Lars Rossing et al. "Bonding between silicones and thermoplastics using 3D printed mechanical interlocking". In: Materials E Design 186 (2020), p. 108254.

[55] Ivan Q Vu et al. "Characterizing the effect of print orientation on interface integrity of multi-material jetting additive manufacturing". In: Additive Manufacturing 22 (2018), pp. 447-461.

[56] Thomas S Lumpe, Jochen Mueller, and Kristina Shea. "Tensile properties of multimaterial interfaces in 3D printed parts". In: Materials $\mathcal{E}$ Design 162 (2019), pp. 19.

[57] Charlie CL Wang and Dinesh Manocha. "GPU-based offset surface computation using point samples". In: Computer-Aided Design 45.2 (2013), pp. 321-330. 